Aus der physiologischen Abteilung der Zoologischen Station zu Neapel und der physiologischen Anstalt zu Innsbruck.

\title{
Histologische Untersuchungen über die Innervation der glatten und der ihr verwandten Muskulatur der Wirbeltiere und Mollusken.
}

\author{
Von
}

Prof. F. B. Hofmann.

Hierzu Tafel XXI.

Die Gruppe der Muskulatur, mit welcher sich diese Abhandlung befasst, begreift in sich Muskeln, welche im ausgebildeten Zustande aus zumeist einkernigen, spindeligen oder verastelten, sternförmig oder sonstwie gestalteten Elementen zusammengesetzt sind, seien diese Elemente nun "längsgestreift", wie in der glatten Muskulatur der Wirbeltiere, doppeltschräggestreift, wie sie in der Mantel- und Flossenmuskulatur der Cephalopoden vorkommen, oder quergestreift, wie der Herzmuskel der Wirbeltiere. Im allgemeinen gehören die hier in Betracht kommenden Muskeln zu jenen, welche die Brüder $\mathrm{Hert}$ wig ${ }^{1}$ ) als mesenchymatöse bezeichnet haben, und ich hätte gerne diesen Ausdruck als bequemen Sammelnamen verwendet, wenn nicht dadurch eine bestimmte Hypothese über ihre Histogenese in die Diskussion hereingetragen würde, die, wenigstens bei Wirbeltieren, nicht für alle glatten Muskeln zutreffen soll. Für den ausgebildeten Zustand wird man aber jedenfalls an der Zugehörigkeit der oben angeführten Muskulatur zu e in er Gruppe festhalten müssen, auch wenn ein passender Name für sie noch nicht gefunden ist. $\mathrm{Zu}$ den allen aufgezählten Muskeln dieser Gruppe gemeinsamen Eigentümlichkeiten gehört nach fremden und eigenen Untersuchungen auch die Art der Innervation, von deren Besonderheiten im folgenden hauptsächlich eine etwas ausfübrlicher besprochen werden soll, auf die ich zuerst bei der Untersuchung der Innervation des Froschherzens aufmerksam wurde. An diesem Objekt

1) Die Coelomtheorie. Jena, Fischer. 1881.

Archiv f. mikrosk. Anat. Bd. 70. 
hatte ich ${ }^{1}$ ) nämlich in Übereinstimmung mit Ranvier ${ }^{2}$, Hey mans und Demoor ${ }^{3}$ ) und anderen gesehen, dass die Muskelzellenzüge allerorts von einem ungemein dichten Nervengeflecht durchsetzt waren, und es fragte sich, ob diese Nervengeflechte nicht vielleicht überall untereinander Anastomosen eingingen, sodass ein wirkliches $\mathrm{N}$ erven $\mathrm{n}$ et $\mathrm{z}$ innerhalb der Muskulatur anzunehmen wäre. $Z$ war liess sich diese Frage damals nicht vollkommen erledigen, aber eines wenigstens konnte, ebenfalls in Übereinstimmung mit Ranvier, gezeigt werden: dass die öftel beschriebenen freien, sogenannten knöpfchenförmigen Enden der Nervenfasern im Herzmuskel Kunstprodukte sind, hervorgerufen durch unvollständige Färbung oder Imprägnation nach Golgi. Je besser die Golgi-Imprägnation gelungen war, desto weniger solcher freier Enden waren zu sehen, und an Stellen mit allerreichster Imprägnation der Nervenfasern fehlten sie vollständig.

$\mathrm{Ob}$ freilich von den letzten noch imprägnierten Nervenästchen nicht vielleicht noch kurze, ihrerseits frei endigende Seitenzweigchen in die Muskelzellen hinein abgegeben werden, welche etwa mit der Golgimethode nicht dargestellt werden können, das liess sich nicht so sicher ausschliessen, wenn es auch sehr zweifelhaft gemacht werden konnte (1. c. S. 103 ff.). Ich komme auf diese Frage weiter unten (S. 379) nochmals zurück. Für die sogleich auszuführende Schlussfolgerung ist sie ohne Belang.

Finden sich nun aber keine freien Enden der Nervenfasern bei reichster Nervenfärbung, so bleiben nur zwei Möglichkeiten übrig: entweder nämlich gehen die durch die Imprägnation sichtbar gemachten Endverzweigungen einer jeden Stammnervenfaser ${ }^{4}$ ) schleifenförmig ineinander über, es bildet also jede Stammnervenfaser für sich abgeschlossene Netze, oder aber die Endästchen

1) Das intrakardiale Nervensystem des Frosches. Hi s' Archiv, 1902, S. 54.

2) Appareils nerveux terminaux des muscles de la vie organique. Paris, 1880.

3) Etude de l'innervation du coeur des vertébrés à l'aide de la méthode de Golgi. Arch. de Biol. XIII, S. 619, 1894.

4) Unter Stammnervenfaser soll hier, wo es sich um gar keine Theorie (der Neuronen oder eine andere) handelt, sondern der feste Boden der Tatsachen nicht verlassen wird, verstanden werden der Nervenfortsatz einer Ganglienzelle vor seiner Aufteilung in die Endäste. Die zur Herzmuskulatur hinziehenden Nervenfasern entspringen beim Frosch zum Teil sicher als gerade Fortsätze der Ganglienzellen des Herzens, offenbar aber, wie sich aus physiologischen Tatsachen ergibt, ausserdem noch als Nervenfortsätze von weiter zentralwärts befindlichen Ganglienzellen. 
der verschiedenen Stammnervenfasern anastomosieren allseitig miteinander, in letzterem Falle also existiert innerhalb der Herzmuskulatur ein durcigehend kontinuierliches, echtes Nervennetz.

Trotzdem ich nun bei meinen Prăparaten, die ich seither auf dem internationalen Physiologenkongresss zu Brüssel demonstriert habe, die allergünstigsten Bedingungen für das Studium dieser Verhăltnisse hatte (Flächenpräparate dünner Membranen - keine Schnitte! - in denen sich die nach der Golgimethode imprägnierten Nervenfasern ausserordentlich scharf vom Grunde abheben, an den gut gelungenen Präparatstellen vollständige Freiheit von Niederschlägen), liess sich doch eine sichere Entscheidung in dieser Alternative nicht făllen. Dazu wäre erforderlich gewesen, dass man die Verzweigung des Nervenfortsatzes einer Ganglienzelle durch die Muskulatur hindurch in eine andere Ganglienzelle hinein hätte verfolgen können, und das war nicht möglich.

Sowie nun aber für den aus Spindelzellen zusammengesetzten Herzmuskel der Amphibien das Fehlen freier Nervenenden nachgewiesen und damit die eben angeführte Alternative aufgeworfen war, erhob sich dieselbe Frage auch für die analog aufgebaute glatte Muskulatur, und schon damals habe ich mir an verschiedenen Objekten bei Wirbeltieren (Gefäss-, Darm-, Blasenmuskulatur) Aufklärung darüber zu verschaffen gesucht, ob sich die am Herzen gewonnenen Resultate auch auf die übrige mesenchymatöse Muskulatur übertragen lassen. Doch führten diese Versuche damals zu keinem entscheidenden Resultate.

In einem andern Sinne hat spater $\mathrm{B}$ ethe, dem meine $\mathrm{Ab}$ handlung entgangen war, in seinem Buche „Allgemeine Anatomie und Physiologie des Nervensystems" (Leipzig, Thieme, 1903) die Existenz kontinuierlicher Nervennetze im Herzmuskel des Frosches behauptet. Bei Bethe ist diese Frage verquickt mit der Annahme, dass die an den Endaushreitungen der marklosen Nervenbündel liegenden Kerne Kerne von Ganglienzellen seien, welch letztere durch netzförmige Anastomosen miteinander verbunden seien. Solche periphere Nervennetze sollen nach Bet he nicht bloss in der Muskulatur des Gefässsystems der Wirbeltiere, sondern insbesondere ausgebreitet auch bei gewissen wirbellosen Tieren, speziell bei Mollusken und Coelenteraten, vorhanden sein. Bei 
den letzteren sollen sie geradezu das ganze Nervensystem ausmachen.

Wie man jetzt schon sieht und wie sich später noch klarer ergeben wird, deckt sich Bethes Darstellung keineswegs mit der meinigen. Immerhin enthielt sie den Hinweis darauf, dass sich diese Fragen vielleicht an der Muskulatur von Wirbellosen leichter entscheiden liessen, als an der Muskulatur der Wirbeltiere und weckte den Wunsch, eine vergleichend-histologischphysiologisehe Studie an diesen vermutlich günstigeren Objekten durchführen zu können. Ein Aufenthalt an der Zoologischen Station zu Neapel zu Ostern 1904, welcher mir durch ein Reisestipendium aus den Mitteln der Albrechtstiftung in Leipzig ermöglicht wurde, bot mir Gelegenheit dazu. ${ }^{1}$ )

Bei der verhăltnismassig kurzen Dauer meines Aufenthaltes in Neapel musste ich selbstverständlich unter den mannigfachen sich darbietenden Versuchsobjekten eine Auswahl treffen. Ich beschränkte mich daher, meiner ganzen Fragestellung entsprechend, von vornherein auf die Untersuchung von "Mesenchymmuskeln“ im Sinne der Brüder Hertwig, und da ich das Glück hatte, sehr bald auf ein Untersuchungsobjekt zu stossen, das der histologischen wie physiologischen Prüfung gleich leicht zugänglich war, nämlich die Chromatophoren der Cephalopoden, so ist es begreiflich, dass ich der Untersuchung derselben hauptsächlich meine Zeit widmete. Daneben berücksichtigte ich die übrige Muskulatur dieser Tiere nur noch soweit, als ich es für die folgende-physiologische Untersuchung brauchte.

\section{Die Innervation der Chromatophoren von Cepha- lopoden, insbesondere von Loligo.}

Die Chromatophoren der Cephalopoden werden gebildet von pigmenthaltigen ein- oder mehrkernigen Zellen, welche unter dem Hauptepithel in den oberflächlichen Schichten des allseits von Muskelzügen durchflochtenen kutanen Bindegewebes liegen. Ihre Histologie und ihre Entwicklung hat in den letzten Jahren

1) Herrn Dr. Lo Bianco bin ich für die reichliche Versorgung mit Tiermaterial, den Herren Dozent Dr. Burian und Dr. Ba uer, dem Leiter und dem zoologischen Beirat der physiologischen Abteilung, für ihre bereitwillige Unterstützung zu besonderem Dank verpflichtet. 
durch die Untersuchnngen von Solger, $\left.{ }^{1}\right)$ Steina $\left.\mathrm{ch},{ }^{2}\right) \mathrm{H}$. Rabl ${ }^{3}$, und $\mathrm{Chun}{ }^{4}$ ) eine ziemlich weitgehende Aufklärung erfahren. In der folgenden orientierenden Einleitung halte ich mich an die Angaben dieser Autoren, die ich, soweit sie für meine Untersuchungen in Betracht kommen, auch aus eigener Erfahrung bestătigen kann. Am Rande des Pigmentklümpchens setzen sich bei der vollentwickelten Chromatophore ringsherum mit verbreiterten Füsschen die sogenannten Radiärfasern an, über deren Natur, ob sie Bindegewebs- oder Muskelfasern seien, lange gestritten wurde. Die Frage ist in der letzten Zeit, insbesondere durch Steinach und $\mathrm{H}$. Rabl, mittels histologischer und physiologischer Beweise wenigstens für die uns hier interessierenden Arten Loligo vulg., Sepia offic., Eledone mosch., Octopus endgültig im letzteren Sinne entschieden worden. Auf Grund der Tatsache, dass Nervenfasern an ihnen endigen, wurden sie auch von Solger und $\mathrm{Chun}$ für Muskelfasern gehalten. Ich kann hinzufügen, dass sie sich auch bei der vitalen Methylenblaufärbung ebenso verhalten wie glatte Muskelfasern, d. h. in solchen Fällen, in welchen Färbung der glatten Muskelzellen auftritt, was ja sehr hăufig geschieht, ohne dass man die Bedingungen dafür recht in der Hand hat, fürben sie sich in sehr schöner Weise ebenfalls blau. ${ }^{5}$ ) Nach einem solchen Methylenblaupräparate ist Fig. 1 auf Taf. XXI angefertigt worden, welche dazu bestimmt ist, die Ansatzverhältnisse der Muskelfasern an den Körper der Chromatophore und die weitgehende Überkreuzung der Radiärmuskeln benachbarter Chromatophoren zum Zwecke des unmittelbaren Vergleichs mit den Innervationsverhaltnissen (Fig. 2) deutlich

1) Zur Kenntnis der Chromatophoren der Cephalopoden und ihrer Adnexa. Dies Arch., Bd. ๖̌3, S. 1, 1899.

2) Studien über die Hautfärbung und über den Farbenwechsel der Cephalopoden. Nebst Versuchen über die autogene Rhythmizität der Chromatophoren-Muskeln. Pflügers Arch. f. Physiol., Bd. 87, S. 1, 1901.

a) Über den Bau und die Entwicklnng der Chromatophoren der Cephalopoden, nebst allgemeinen Bemerkungen über die Haut dieser Tiere. Sitzungsbericht d. Wiener Akad. d. Wiss., Mathem.-naturwiss. Klasse, Bd. 109, Abt. 3, S. $341,1900$.

*) Übẹr die Natur und die Entwicklung der Chromatophoren bei den Cephalopoden. Verhandl. d. deutschen zoolog. Geselssch. 1902, S. 162.

5) Eine solche, aber unvollständige "Imbibition" der Radiärfasern mit dem Farbstoff hat schon Solger beobachtet. 
zu machen. "Jede der Radiärfasern besteht aus einer protoplasmatischen Masse, mit einem gegen das basale der Chromatophore zugewandten Ende hin verschobenen Kern. Diese Masse wird von einer ......... stark lichtbrechenden Wand umfasst. An der Insertionsstelle des Muskels an die Chromatophore zeigt sich, dem Kern entsprechend, eine leichte konische Anschwellung" ... (Solger, 1. c. S. 11). Die stark lichtbrechende Substanz der Radiärfasern ist aber nicht ganz homogen, sondern besitzt nach Stein ach (1. c. S. 8) eine echte fibrilläre Lăngsstreifung. Die Radiärfasern setzen sich nicht voneinander isoliert an den Pigmentkörper an, vielmehr gehen ibre verbreiterten konischen Endeh mit denen der benachbarten Fasern Verbindungen ein, welche sich Farbstoffen gegenüber ebenso verhalten, wie die Muskelfasern selbst. Durch diese Verbindung sämtlicher Radiärfasern einer Chromatophore, entsteht eine zusammenhängende muskulöse Zone, welche dicht anliegend den Äquator der Pigmentplatte gürtelartig umspannt und auch auf deren Ober- und Unterfläche etwas übergreift" (Steinach l. c. S. 9). Am distalen, vom Pigmentkörper der Chromatophore abgewandten Ende verschmälern und verästeln sich die Radiärmuskeln, und ihre Enden gehen, wie sich bei näherer Betrachtung zeigt, in Bündel von Hautmuskelfasern über, welche die Haut allenthalben durchzieben. „Es hat oft den Anschein, dass eine Verschmelzung stattfinde ..., aber als ganz sicher kann in diesen Fällen angenommen werden, dass eine innige Anlehnung der Radiärfasern an die Hautmuskulatur besteht (Steinach, 1. c. S. 11). Ich füge hinzu, dass man auch bei Methylenblaufärbung mitunter die büschelförmige Verästelung der Radiärfasern beobachtet, wobei sie isoliert gefärbt sind, wahrend die Hautmuskelzellen, an welche sie sich anlegen, ungefarbt bleiben. Es ist dies dasselbe Verhältnis. das man auch sonst bei der vitalen Methylenblaufärbung glatter Muskeln findet. So kann man z. B. auch in der Mantel- und Flossenmuskulatur von Cephalopoden diese isolierte Färbung einzelner Muskelzellen beobachten, während die dicht daneben liegenden ganz ungefarbt bleiben. Ebenso verbält es sich mit den spindeligen oder verästelten nebeneinander liegenden Elementen des Herzmuskels der Amphibien (auch im Vorhofe des Herzens von Octopus habe ich dasselbe gesehen). Bezüglich weiterer Details über die Verăstelung des distalen Endes der 
Radiärfasern muss ich auf Steinachs Darlegungen verweisen. Nach diesen und nach eigenen gelegentlichen Beobachtungen möchte ich glauben, dass bezüglich der Endverästelungen starke Variationen vorhanden sind. Aus solchen Verschiedenheiten, je nach der Spezies, ist es wohl auch zu erklären, dass $\mathrm{Ch}$ u n bei Bolitaena keine distalen Verästelungen der Radiärmuskeln auffand.

Sebr beachtenswert für die physiologische Untersuchung ist die ausserordentlich reichliche Überkreuzung der Radiärmuskeln benachbarter Chromatophoren. So überkreuzen sich in der Mitte der Fig. 1 auf Taf. XXI die Radiärmuskeln von vier Chromatophoren. Diese Verfilzung kann, wie Stein ach ausführt, leicht zu Täuschungen Veranlassung geben, indem Radiärmuskeln benachbarter Chromatophoren miteinander zu verschmelzen scheinen. während sie in Wirklichkeit übereinander wegziehen. Mir ist aufgefallen, dass die Lănge der Radiärfasern bei den verschiedenen nebeneinander liegenden Chromatophoren sehr variiert, wie dies schon Fig. 1 zeigt. Bei Sepia und Loligo finden sich insbesondere einzelne dunkle Chromatophoren mit sehr langen Radiärmuskeln, welche ziemlich vereinzelt mitten zwischen kurzfaserigen darinnen liegen.

Die Innervation der Chromatophoren ist mit Hilfe der neueren Nervenfärbungsmethoden von $J_{0}$ u b i i ${ }^{1}$ ) und $S$ olger (l. c.) untersucht worden. Samassa ${ }^{2}$ ) hat die Nervenversorgung der Chromatophoren an mit Hämatoxylin gefärbten Präparạten, $\mathrm{Ph}$ is ali ${ }^{3}$ ) an Pikrokarminpraparaten, $\mathrm{Ch}$ un (l. c.) neuerdings bei einer durchsichtigen Tiefseeform (Bolitaena) an FlemmingPräparaten ohne jede Färbung beobachtet. Ich möchte die älteren Angaben von $\mathrm{Joubin}$ und $\mathrm{Ph}$ is alix hier nicht weiter reproduzieren, sondern verweise diesbezüglich auf die eingehende Kritik in der Abhandlung von Solger; Samassa sah (bei Loligo marmorata) bloss ein Herantreten von Nervenfasern an den Chromatophorenkörper, nicht aber an die Radiärfasern.

Von den neueren Abhandlungen sind sehr wichtig zunächst die Angaben von Solger. Er sah bei der vitalen Methylenblau-

1) Recherches sur la coloration de tégument chez les Céphalopodes. Arch. de zool. expér., 2 me série, 10, p. 277, 1892.

2) Bemerkungen über die Chromatophoren der Kephalopoden. Verh. d. naturwiss.-mediz. Vereins z. Heidelberg (2), 5, S. 133, 1893.

3) Structure et développement des chromatophores chez les Céphalopodes. Arch. de physiul. norm. et pathol., כ̃ me série, vol. 4, p. 445, 1892. 
färbung an den Radiärmuskeln (von Rossia makrosoma, Loligo vulgaris und Sepiola Rondeletii) in der Năhe des Chromatophorenkörpers variköse Linien oder Körnchenreihen. Die Körnchen waren entweder kugelig oder es zeigten sich Figuren von komma-, haken- oder schlingenförmiger Gestalt, was darauf hindeutete, dass die hier teilweise gefarbte Nervenfaser geschlängelt verlief. Die so gestalteten Körnchenreihen oder varikösen Fäden hörten zumeist in einiger Entfernung vom Pigmentkörper auf. Bezüglich der eigentlichen Endigungen spricht sich Solger sehr zurückhaltend aus: „Ich lasse übrigens .... die Frage ausdrücklich noch offen, ob es mir bei den von mir ausgeführten Methylenblauversuchen gelungen ist, die Nervenfädchen nun auch bis zu ihrer allerletzten Endigung zu verfolgen."

Die Beobachtungen von $\mathrm{Chun}$ über Nervenendigungen an ungefärbten Prăparaten beziehen sich zunăchst auf Entwicklungsstadien von Chromatophoren. Sobald die Radiărasern bei ihrem Auswachsen den Durchmesser der Zelle um das zwei- bis dreifache an Länge übertreffen, kommen sie in Kontakt mit feinen Nervenstämmchen der Haut, und dann bilden sich Nervenendjgungen an den Radiärmuskeln aus, weiche die denkbar verschiedenartigsten Bilder zeigen. „Am hăufigsten kommt vor, dass das Ende einer Radiärfaser an ein Nervenstämmchen stösst und nunmehr breit in dasselbe übergeht. Eine scharfe Grenze lasst sich in dieser meist dreieckig gestalteten kleinen Endplatte weder für die Radiărfaser noch für den Nerven nachweisen." Es können aber auch zwei, ja selbst drei Radiärfasern an dasselbe Nervenstämmchen stossen und jedesmal sich leicht verbreiternd mit demselben verschmelzen. Als wesentlichen Punkt seiner Befunde hebt $\mathrm{Chun}$ hervor, dass ,einerseits ausschliesslicb eine Innervierung der Radiärfasern, niemals aber eine solche des Zellkörpers $\mathrm{zu}$ beobachten ist, und dass andererseits auch nicht eine einzige Chromatophore anzutreffen war, bei der nicht wenigstens ein Ausläufer mit einem Nerven in Verbindung gestanden hätte " (l. c. S. 176).

An den Fasern voll entwickelter Chromatophoren sind die Nervenendigungen „durchaus nicht so leicht wabrzunehmen, wie an den jüngeren Stadien. Nur seiten vermag man mit aller Scharfe nachzuweisen, dass ein über sie hinweggleitendes Nervenstämmchen auch tatsächlich eine Verbindung mit der unterliegenden 
Faser eingeht". Diese Verhältnisse bei den entwickelten Chromatophoren werden von $\mathrm{Chu}$ n durch eine Textfigur (Fig. 3, S. 170) illustriert, in welcher ein stärkerer Nervenast abgebildet ist, „der mit seinen Verzweigungen ein Endnetz bildet". "An der Stelle, wo die feineren Äste ${ }^{*}$ eines solchen Nervenstämmchens, sich gabeln, ist eine gewöhnlich dreieckig gestaltete plattenförmige Verbreiterung ausgebildet, in der die ovalen, fein granulierten Kerne, bisweilen aber auch homogene, stark lichtbrechende Kerne, gelegen sind" (l. c. S. 171).

Resumieren wir von all diesen Angaben das, was für die folgende Darstellung hauptsächlich in Betracht kommt, so sprechen Samassa, $\mathrm{Ph}$ is alix and $\mathrm{J} o \mathrm{ub}$ in von Endigungen der Nerven am Körper der Chromatophoren, was aber von den späteren Untersuchungen nicht bestatigt wurde. Solger sah an den RadiärmuskeIn gerade oder geschlängelt verlaufende Nervenfasern, deren Färbung meist vor dem Pigmentkörper der Chromatophoren aufhörte, über deren eigentliche Endigung er aber nichts aussagen konnte. Auch $\mathrm{Chun}$ sah jedenfalls das Herantreten von Nervenfasern an die Radiarmuskeln und an der Vereinigungsstelle eine so innige Aneinanderlagerung, dass eine Grenze zwischen Nerv und Muskel am ungefärbten Prapparate nicht mehr wahrzunehmen war. Ausserdem bildet $\mathrm{Chun}$ noch ein peripheres Nervennetz mit Kernen an den Verzweigungsstellen $\mathrm{ab}$.

Für meine eigenen histologischen Untersuchungen, die mit der vitalen Methylenblaumethode ausgeführt wurden, erwies sich als. das geeignetste Objekt die Haut von Loligo vulgaris. An den Chromatophoren von Sepia officinalis bekommt man zwar bei der vitalen Methylenblaubehandlung auch manchmal Nervenfürbungen, aber nie so gut wie bei der erstgenannten Spezies. Die Herstellung der Präparate ist die denkbar einfacbste. Unmittelbar nach dem Tode des Tieres zieht man die Haut in grossen Stücken ab und wirft sie in eine verdünnte Lösung von Ehrlichs rektifiziertem Methylenblau in Seewasser. ${ }^{1}$ ) Nach

1) Leider habe ich auf die genaue Feststellung der Konzentration der Lösung (etwa ${ }^{1 / 60}{ }^{0} \%$ ) kein besonderes Augenmerk gerichtet, sondern mich an meine Erfahrung über die für andere Objekte günstige Farbe der Lösung gehalten. Übrigens hängt das Gelingen der Fürbung wohl auch nicht so sehr von der Konzentration der Lösung, als vielmehr vom mehr oder weniger frischen Zustand des Objektes ab. 
20-30 Minuten nimmt man ein Hautstück aus der Lösung, breitet es auf einem Objektträger aus und sieht nach, ob sich an der Luft allmăhlich die Nerven fărben. Sobald man auf diese Weise durch Probieren den richtigen Zeitpunkt ermittelt hat, breitet man nunmehr alle Stücke auf Glasplatten an der Luft aus und wartet $a b$, bis die Nerven sich intensiv blau gefärbt haben. Da der Luftsauerstoff in die Haut rasch eindringt, bekommt man die Färbung bald. Die Schwierigkeiten beginnen erst beim Versuche, die Färbung zu fixieren. Nach der Methode von Bethe durch Behandeln mit Ammoniummolybdat in der Kälte habe ich viele Präparate verloren. Es erwies sich daher immer noch am zweckmässigsten, nach der Methode von Arnstein und Dogiel mit Ammoniumpikrat zu fixieren und die.Prapparate sodann in einem Gemisch von Glyzerin und gesăttigter Ammoniumpikratlösung zu gleichen Teilen aufzuhellen. Leider haben sich auch so meine Präparate nicht alle gleich gut gehalten, sondern einige, von denen ich Zeichnungen schon auf dem internationalen Physiologenkongress zu Brïssel im September 1904 demonstriert habe, sind inzwischen stark abgeblasst. Andere Prăparate sind dagegen jetzt noch gut erhalten und es besteht daher die Hoffnung, dass sie es noch längere Zeit bleiben.

Wenn man an einem gut gelungenen Methylenblaupräparate mit schwacher Vergrösserung auf die Chromatophorenschicht der Haut einstellt, so ist man zunächst überrascht von dem verwirrenden Reichtum an feinsten Nervenfasern (vergl. Fig. 2 auf Taf. XXI), der dem in der Herzmuskulatur der Amphibien in keiner Weise nachsteht, und unwillkürlich erhebt sich da die Frage, ob denn das wirklich alles Nervenfasern sind. Wir wissen ja durch S. Ma y e r, ${ }^{1}$ ) was sich alles mittels der "vitalen " Methylenblaumethode färben lässt, und es ist besonders darauf hinzuweisen, dass, abgesehen vom Bindegewebe, sehr baufig sich auch glatte Muskelfasern fărben, und dass deren Verästelungen oft tăuschend åhnliche Bilder liefern wie die Nervenfasern. Der erwăhnte Zwerfel liess sich nun in meinen Prăparaten - ganz abgesehen von dem charaliteristisch varikösen Aussehen der nervösen Endverästelungen - vollkommen sicher beseitigen, weil an verschiedenen Stellen nicht bloss die Endverzweigungen, sondern

1) Beiträge zur histologischen Technik. I. Die Methode der Methylenblanfärbung. Zeitschrift f. wiss. Mikrosk., Bd. 6, S. 422, 1889. 
auch die dickeren marklosen Nervenfasern gefärbt waren und weil sich hier ganz unzweifelhaft der Ursprung der Endästchen aus den grösseren Nervenfasern feststellen liess (vergl. Fig. 3 auf Taf. XXI). Betrachtet man die dickeren Nervenfasern bei sehr starker Vergrösserung (Immersion), so nimmt man an manchen Stellen mehr oder weniger deutlich eine feine, wellige Längsstreifung wahr, als Ausdruck der fibrillären Struktur derselben. Eine scharfe Differenzierung der einzelnen Fibrillen aber, das ist für spătere Überlegungen zu konstatieren wichtig, ist in meinen Methylenblaupräparaten nicht vorhanden, es ist ausser den Fibrillen auch die $\mathrm{Z}$ wischensubstanz mitgefärbt.

Die dickeren Nervenfasern der Stämmchen verzweigen sich auf ganz ausserordentlich weite Strecken hin, und sie bilden mit ihren Teilästen ein Geflecht, von welchem die Teiläste $b, c$ und $d$ der Fig. 3 auf Taf. XXI nur eine sehr schwache Vorstellung geben. Denn hier sind nur zwei Nervenfasern (eine breite und eine feine) aus dem ganzen Nervenbündel gefärbt, wăhrend sonst mehrfach eine ganze Anzahl von breiten, zu einem solchen Geflecht vereinigten Nervenfasern gefärbt sind. Wir können diesen Plexus, der $\mathrm{zumeist}$ von breiteren, sich vielfach teilenden, nicht varikösen Nervenfasern gebildet wird, nach Analogie mit den bekannten Verhältnissen in der glatten Muskulatur der Wirbeltiere als den Grundplexus bezeichnen. Seine Anordnung steht in keiner nachweisbaren Beziehung zur Anordnung der Muskelfaserzüge. Am stärksten entwickelt ist er in der Gegend der Einstrahlung der Chromatophorennerven in die Haut, und er stellt demnach die allmähliche Aufteilung und Ausbreitung der Nerven innerhalb ihres Innervationsgebietes dar (vergl. darüber den physiologischen Teil dieser Untersuchungen, der gleichzeitig in Pflügers Archiv erscheint).

Von diesem Grundplexus gehen nun als kollaterale Seitenabzweigungen stärkerer, oder als Endverzweigung feiner schon im Grundplexus enthaltener Nervenfäserchen zarte Fädchen $a b$, welche ebenfalls sehr häufig zu Bündelchen vereinigt sind. Diese bestehen aber diesmal nur aus feinen, zumeist varikösen Nervenfäserchen, die sich in vollstăndig wirrer Weise durcheinander flechten. Hat man günstige Stellen des Präparats, so sieht man bei starker Vergrösserung und enger Blende, dass diese Bündelchen variköser feinster Nervenfasern an Bündeln von Hautmuskelfasern 
entlang laufen, stellenweise auch zwischen dieselben eindringen. Diese Nervenfäserchen geben also in ihrem Verlauf die Anordnung der Muskulatur wieder, und wir müssen sie demnach als das Analogon jener Geflechte betrachten, welche in der glatten und der ihr verwandten Muskulatur der Wirbeltiere als intramuskulärer Plexus bezeichnet wird. Ziehen wir z. B. die Verbältnisse im Amphibienherzen zum Vergleich heran, so unterscheidet sich auch dort der intramuskuläre Plexus vom Grundplexus durch das charakteristisch verschiedene Verhalten der Verlaufsrichtung der Nerven: „Während die Richtung der Bündel im Grundplexus im allgemeinen unabhängig ist von der Anordnung der Muskulatur, halten die Fäden des intramuskulären Plexus im ganzen und grossen die Richtung der Muskelbündel ein, indem sie lange Strecken den einzelnen Muskelzellen parallel laufen, wobei sie allerdings gelegentlich umbiegen oder seitlich abschwenken, um sich zwischen andere Muskelzellen einzuschieben, oder gar auf benachbarte oder kreuzende Muskelbündel überzugehen " (S. 95 meiner oben S. 362 zitierten Abhandlung). Ich füge hier berichtigend hinzu, dass sogar schon die letzten Worte bloss für jene oberflächlichsten Nervenfasern zutreffen, welche Gerlach als perimuskulären Plexus bezeichnet hat und die man eigentlich noch als allerletzte Verzweigung des Grundplexus auffassen muss, sodass also die Differenz sogar noch schärfer zu formulieren ist, als ich es früher getan habe.

Nur ein Unterschied ist mir beim Vergleich der beiden Objekte aufgefallen. An den Hautmuskelzügen der Cephalopoden finde ich die Nervenbündel mehr oberflächlich den Muskelzügen anliegend, während die Herzmuskelzüge durch und durch von Nervenfädchen durchsetzt waren. Indessen beruht dieser Unterschied vielleicht anf der Verschiedenheit der Untersuchungsmethoden. Die Golgi-Imprägnation bringt die Nervenfasern in der ganzen Dicke des Muskelzugs gleichmässig zur Anschauung, während die Methylenblaufarbung auch schon beim Froschberzen hauptsăchlich die oberflächlichen Nerven betrifft, in viel geringerem Grade die etwas tiefer liegenden. Wenn es also vielleicht nur der Mangelhaftigkeit der Methode zuzuschreiben ist, dass die Nerven an den Hautmuskelzügen der Cephalopoden nicht intramuskulär, sondern mehr oberflăchlich, perimuskulär, erscheinen, so möchte ich doch vorläufig, bis die Sache auf andere Weise 
geklärt ist, den Ausdruck „intramuskulärer Plexus“ vermeiden und bloss von einem Endplexus sprechen.

Es ist keineswegs immer leicht, die Grenze zwischen Grundplexus und Endplexus festzustellen, wenn man sich nicht an das eben gegebene Kriterium der Übereinstimmung des Verlaufs von Nerv und Muskel halten kann. Denn es ist zu berücksichtigen, dass der Grundplexus an jenen Hautstellen, welche von der Einstrahlung der Hautnerven am entferntesten liegen, infolge der häufigen Teilung der Nervenfasern ebenfalls nur noch aus feinen Fadchen zusammengesetzt ist. Nun sind zwar die Fasern des Endplexus zumeist melur oder weniger varikös, sehr selten auf längere Strecken hin ganz glatt, während dem Grundplexus wohl variköse Fädchen beigemischt sein können, die glatten Fasern dagegen die überwiegende Mehrzahl bilden. Als durchgreifendes Unterscheidungsmerlimal kann man aber die Varikosität der Nervenfasern nicht benützen. Übrigens besteht auch hierin eine Ähnlichkeit mit den Nerven des Froschherzens insofern, als die Varikositäten dort ebentalls am häufigsten die Fäden des intramuskularen Plexus betrafen, eine ganz allgemeine Regel darïber sich aber auch dort nicht aufstellen liess (vergl. dazu meine auf S. 362 zitierte Abhandlung, S. 106, Anm. 2).

Infolge des grossen Reichtums der Cephalopodenhaut an sie kreuz und quer durchflechtenden Muskelzügen bringt nun ein Präparat, in welchem die Nervenfasern des Endplexus mitgefärbt sind, mit schwacher Vergrösserung die schon erwähnte verwirrende Menge feinster Nervenfasern zur Ansicht, die von Fig. 2 auf Tafel XXI einigermaßen wiedergegeben wird. Aus diesem Gewirr treten aber diejenigen Nervenfasern, welche die Radiärmuskeln der Chromatophoren begleiten, infolge ihrer charakteristischen Anordnung kräftig hervor. Sehen wir in dem Übersichtsbild der Fig. 2 von allem anderen $a b$ und fassen nur die in der Mitte liegende Chromatophore ins Auge, so erkennen wir, besonders beim Vergleich mit Fig. 1, dass jede Radiärmuskelfaser in ihrer ganzen Länge begleitet ist von mindestens e in e m Nervenfaden, so dass man aus dem blossen Bilde der Nervenfärbung mit grosser Genauigkeit die Anordnung der Radiărmuskeln wiedererkennen kann. Die Nervenfasern verlaufen hier, wo die Chromatophore nicht expandiert ist, die Muskelfasern also nicht kontrahiert sind, ziemlich geradlinig, sehr wenig ge- 
schlängelt. An stark expandierten Chromatophoren, deren Muskelfasern also im Kontraktionszustand fixiert sind, sind sie dagegen oft sehr stark geschlängelt (vergl. Fig. 3 auf Taf. XXI, Stelle e). In der Nahe des Körpers der Chromatophore, in der Gegend ungefähr, wo sich die Muskelkerne befinden, pflegt die Färbung der die Radiarfasern begleitenden Nerven sehr blass zu werden und zumeist, besonders in den weniger gelungenen Präparaten, ganz aufzuhören. In solchen Fällen, insbesondere auch, wenn das Endstück stark geschlängelt ist (wie an Stelle e in Fig. 3), erkennt man sofort die von Solger abgebildeten Verhaltnisse wieder, nur dass hier die Nervenfaden in ihrer Kontinuität. nicht wie in den Prăparaten von Solger nur in kurzen Stücken, gefärbt sind. In dieser Beziehung liefern also meine Präparate die völlige Bestätigung der Feststellungen von Solger. In mehreren Punkten aber gehen sie darüber hinaus. Ist nämlich die Färbung der Präparate besonders gut, so sieht man, dass das Aufhören der Färbung am Cbromatophorenkörper keineswegs auf eine Endigung der Nervenfasern $\mathrm{zu}$ beziehen ist. Vielmehr lassen sich die Chromatophorennerven an den besten Präparaten längs der Radiarfasern bis an den Chromatophorenkörper heran verfolgen, und dort biegen sie nach der Seite ab und verbinden sich guirlandenförmig mit den Fasern der benachbarten Radiärfasern. Dabei geben sie genau die Form der Einbuchtungen zwischen den einzelnen Radiärmuskelansătzen wieder (man vergl. miteinander Fig. 1 und 2, sowie Fig. 4). Es kann ganz gut an einer und derselben Chromatophore die Färbung an einer Stelle der Umgebung vollständiger sein als an anderen. So zeigt Fig. 2 alle Übergänge von vollständiger Färbung mit geschlossener Guirlande bis zum gänzlichen Aufhören der Fürbung, bevor der Chromatophorenkörper erreicht ist. Für den, welcher das kapriziöse Verbalten der Methylenblaufärbung kennt, wird die Beurteilung der Sachlage keine Schwierigkeiten haben. Es sind offenbar in der Nähe des Chromatophorenkörpers besonders schlechte Bedingungen für das Zustandekommen der Färbung gegeben. Die Folge davon ist, dass man nur ausnahmsweise vollständigere Färbungen erhält, in der Regel dagegen die Farbung ausbleibt. Unter diesen Umständen hat man sich einzig an die bestgelungenen Präparate mit möglichst vollständiger Fărbung zu halten, und diese zeigen eben, dass die 
die Radiärfasern begleitenden Nervenfadchen an der Chromatophore kein Ende finden, sondern schlingenförmig in die benachbarten Nervenfäden hineinlaufen. Die bisher dargestellten freien Enden dieser Nerven sind also Kunstprodulite.

Betrachten wir nun diese Präparate mit starker Vergrösserung, so erkennen wir zunächst die jetzt sichtbare Längsstreifung der Radiärfasern und sehen, dass die Nervenfasern stets in unmittelbarer Berührung an ihnen entlang laufen. Auch die guirlandenförmigen Schleifen liegen der längsgestreiften Muskelsubstanz dicht an, wobei die Langsstreifung an diesen Stellen in derselben Weise wie die Nervenschlingen bogenförmig mit der Konkavität nach aussen von einer Radiärmuskelfaser zur andern hinstreicht (vergl. Fig. 4 auf Taf. XXI).

Solger bildet in;Fig. 3 seiner Abhandlung die Nervenschlingen teilweisn in einiger Entfernung von der Kontur der Radiärfaser ab. Ich habe das Abbiegen einer einzigen kurzen Nervenschlängelung von der Radiärfaser nur einmal beobachtet, sonst stets dichtes Anliegen, wie dies auch Solge r in Fig. $4 \mathrm{~A}$ und $\mathrm{B}$ wiedergibt.

Die Nervenfasern selbst bieten nun bei dieser starken Vergrösserung ein höchst merkwürdiges Bild: Im ganzen präsentieren sie sich als blassviolette Stränge, in denen stellenweise auf kürzere oder längere Strecken hin eine ungemein mannigfaltig gekräuselte. scharf dunkel gefärbte Linie auftritt (vergl. Fig. 4). Man hat mitunter direkt den Eindruck, als ob sich ein ausserordentlich verschlungener feiner Faden in einer blass gefärbten Grundsubstanz befände, und man kommt so zunächst auf den Gedanken, dass man hier eine intensiv tingierte Neurofibrille innerhalb einer protoplasmatischen Grundmasse vor sich habe. Das war eine Zeit lang auch meine Meinung und ich habe sie auf der Meraner Naturforscherversammlung bei einer Diskussionsbemerkung auch öffentlich ausgesprochen. ${ }^{1}$ ) Ein genaueres Studium der Präparate hat mich indes darüber belehrt, dass diese Auffassung falsch war. Bei Verwendung stärkster Vergrösserungen sieht man nämlich, wie schon angedeutet wurde, an besonders gut gefärbten Stellen, dass dieser Faden keineswegs kontinuierlich durch die blass gefärbte Grundmasse, wenn auch noch so geschlängelt, weiterzieht, sondern dass er nur von Stelle zu Stelle auftritt (Fig. 5 auf Taf. XXI). Sehr häufig erweist sich der dunkle Strich als Begrenzung eines kleinen Knöpfchens, und wenn man genau

1) Verhandl. d. Naturf.-Vers. zu Meran, 190ó, 2. Teil, 2. Hälfte, S. 418. 
zusiebt, so löst sich die blass gefärbte Grundmasse überhaupt in eine Summe von aneinander gereihten violetten Tröpfchen auf, auf oder neben denen vorüber oder auch zwischen denen bindurch manchmal ein fädiges Gebilde zieht. An manchen Stellen sieht man auch zwei solche scharfe Fäden nebeneinander von einer Tröpfchenmasse zur nächsten ziehen.

Die Lösung des Problems ergibt sich, wenn man den Nerven nach dem distalen Ende der Radiărfaser hin verfolgt. Früher oder später sieht man dann, dass die vorher so wirre Masse von Tröpfchen und Strichen sich auflöst in ein Bündel von drei, vier oder mehr einzelnen scharfen Fadchen, welche stellenweise unterbrochen sind durch blasser gefärbte Varikositäten, die eventuell noch einen scharfen Kontur besitzen, der wie ein Faden aussehen kann. Man vergleiche dazu Fig. 6 auf Tafel XXI, wo eine distalere Stelle des in Fig. 5 dargestellten Nervenbündels wiedergegeben ist. Es besteht demnach eine solche scheinbar einheitliche Nervenfaser in der Nuhe des Chromatophorenkörpers aus dem Zusammentreffen einer Anzahl stark variköser Fasern, die man nur an veleinzelten Stellen, wo sie etwas weiter voneinander entfernt sind, gesondert sehen kann, während sie sonst ein ausserordentlich kompliziertes Gesamtbild geben, das sich aus den blass gefärbten Varikositäten und den dunkler gefärbten nicht varikösen Partien der einzelnen im Bündel eng zusammenliegenden Nervenfädchen unentwirrbar zusammensetzt. Dass dies wirklich richtig ist, elgibt sich ferner aus der Beobachtung anderer Radiarfasern mit spärlicherer Nervenfärbung, wo es vorkommen kann, dass tatsächlich bloss eine einzige Nervenfaser von dem Charakter der aufgesplitterten Einzelfädchen der eben beschriebenen Faserbündel an der Muskelfaser entlang zieht. Dann hat man nicht das komplizierte eben geschilderte Bild, sondern einen einfachen scharfen Faden, der von Stelle zu Stelle in etwas schwächer gefärbte Varikositäten übergeht, wie gesagt, dasselbe, was man in Fig. $\dot{b}$ an jedem einzelnen Faden des Bündels auch beobachten kann. Man kann demnach sehr wohl bei starker Vergrösserung einzelne Nervenfasern von einem derartigen Bündel unterscheiden, aber es ist nicht möglich, in einem solchen Bündel die einzelnen Nervenfadchen isoliert $\mathrm{zu}$ verfolgen.

Diese dichte Aneinanderlagerung mehrerer Nervenfädchen, sodass der Eindruck eines einzigen Nerven erweckt wird, ist mir freilich, trotzdem ich 
mich anfangs habe täuschen lassen, nichts Neues. Dasselbe habe ich schon am Froschherzen von den Fasern des intramuskulären Plexus beschrieben (1. c. S. 100): Immer und immer wieder wird man darauf stossen, „dass zwei Nervenfasern sich eine Strecke weit so eng aneinander legen, dass man sie nur gelegentlich kleinerer Biegungen voneinander sondern kann." Dort waren es allerdings Golgipräparate, die ich daraufhin durchmusterte, und diese bieten, vorausgesetzt, dass sie rein sind, der Deutung keine solchen Schwierigkeiten wie die Methylenblaupräparate, weil die Imprägnation eine mehr gleichmässige Schwärzung bedingt, kein solches Abwechseln blasser und stärker gefärbter Partien, wie die Methylenblaufärbung.

Wenn man nun aber weiss, dass eine solche scheinbar einfache Nervenfaser, welche an der Radiärfaser entlang zieht, in Wirklichkeit ein Bündel von mehreren Finzelfädchen darstellt, so wird man auch mit einem ganz anderen Urteil an die Verzweigung dieser Nerven in der Nähe des Chromatophorenkörpers herangehen. Wenn wir hier nämlich eine Teilung einer solchen nur scheinbar einfachen Nervenfaser finden, so ist es damit keineswegs ausgemacht, dass ihr nun auch wirklich die Teilung einer Nervenfaser zugrunde liegt, sondern es ist ebensowohl möglich, dass es sich dabei nur um ein Abschwenken einer vorher im Bündel enthaltenen Einzelfaser handelt. Danach ist also z. B. der Fall zu beurteilen, dass von dem eine Radiärfaser begleitenden Nervenzug Schlingen zu beiden benachbarten Radiärfasern abgehen. Ich habe dies übrigens nicht oft gesehen (ob bloss wegen ungenügender Nervenfärbung, ist fraglich). Ebenso steht es mit dem häufigen Vorkommen von kurzen Reiserchen, welche von einem Nervenzug abzweigen, noch eine kurze Strecke an der Radiärfaser entlang laufen und dann entweder ganz allmählich blasser gefärbt sind und schliesslich sich von der Umgebung nicht mehr unterscheiden, oder deren Färbung ganz plötzlich abbricht. Dass wir es hier mit der wirklichen Endigung einer Nervenfaser oder des Teilastes einer solchen zu tun haben, ist ausserordentlich unwahrscheinlich. In dem ersterwähnten Falle, wenn die Färbung immer blässer wird (wie in Fig. 4 auf Taf. XXI an der Stelle c), ist das eigentlich ganz selbstverständlich. Streiten könnte man nur in dem $\mathrm{z}$ weiten Falle, wie er $\mathrm{z}$. B. durch die Stellen d derselben Figur repräsentiert wird. Wenn man sich aber vergegenwärtigt, wie häufig das Abbrechen der Färbung in der Nähe des Chromatophorenkörpers ist, so wird man es gewiss auch bier für viel wahrscheinlicher halten, dass die Faser d in unserem Falle ebenfalls weiter zieht, und nach Analogie mit 
den vollständigeren Färbungen in der Umgebung wird man wohl annehmen können, dass die Enden $c$ und d nichts anderes sind als eine unvollständig gefärbte Schleife zwischen den Nervenzügen der beiden benachbarten Radiarfasern. So aufgefasst, liefert diese Stelle auch ein Beispiel für den erst besprochenen selteneren Fall der Abschwenkung eines Nervenfaserzuges zu beiden benachbarten Radiärfasern. Sozusagen das Gegenbild dazu findet sich an Stelle $f$ derselben Figur, wo die Schleife gefärbt ist, nicht aber die Fortsetzung derselben an der Faser, welche vermutlich zum Nervenzug e hinüberführte. Hier wird wohl niemand daran zweifeln, dass das Ende bei f keinem Aufhören der Nervenfasern, sondern bloss einem noch dazu ganz allmählichen Aufhören der Färbung entspricht.

Immerhin, selbst wenn man einen solchen Schluss für viele Falle zulässt, für alle lässt er sich nicht beweisen, und man muss daher immer noch eine Möglichkeit ins Auge fassen, welch€ das schleifenförmige Umbiegen der Nervenfasern am Chromatophorenkörper mit dem Vorhandensein freier Nervenenden in Übereinstimmung zu bringen gestattet Es könnte nämlich sein, dass sich die Schleifen der benachbarten Radiärnerven (so will ich die die Radiärmuskeln begleitenden Nervenbündel kurz nennen) am Chromatophorenkörper bloss durchflechten und dass einzelne Fädchen eines Radiärnervenbündels erst an den benachbarten Radiärmuskeln in der Nähe oder in einiger Entfernung vom Chromatophorenkörper frei endigen. Ausschliessen lässt sich diese Annahme nur dann, wenn man Fälle findet, in welchen an beiden Radiärmuskeln wirklich nur eine einzige Nervenfaser'klar verfolgt werden kann. Ich besitze nun in der Tat einige wenige solche Stellen in meinen Präparaten, in welchen dies mindestens in der Nahe des Chromatophorenkörpers der Fall ist. Ich will ganz offen lassen, ob hier wirklich nur eine einzige Nervenfaser an den Radiärmuskeln vorhanden war, gefärbt war nur eine Faser, und diese zeigt auch das schlingenförmige Umbiegen und zieht in der Tat, ohne $\mathrm{zu}$ endigen, wieder von der Chromatophore weg. Das ist entscheidend, und wir können es daher als sichergestellte Tatsache betrachten, dass in Fällen sehr guter Färbung die Radiärnervenfasern nicht in der Nähe des Chromatophorenkörpers endigen, sondern wenn sie hier angelangt sind, wieder umbiegen und am nächsten Radiärmuskel entlang zurück- 
laufen. Dass sich alle Radiärnervenfasern so verhalten, lässt sich natürlich daraus nur mit grosser Wahrscheinlichkeit erschliessen, nicht direkt erweisen. Möglich, wenn auch äusserst unwahrscheinlich wäre es schon, dass ausser den schleifenförmig umkehrenden Nervenfasern auch noch andere vorhanden sind, welche wirklich endigen. Ich selbst möchte mich freilich nicht zu dieser Annahme entschliessen, denn dies würde doch ganz aus der Analogie herausfallen. Wir müssen uns erinnern, dass das Resultat, das wir hier infolge der bekannten Mängel der Methylenblaumethode nur für einige Fälle ad oculus demonstrieren konnten, in der Herzmuskulatur der Amphibien mit viel grösserer Allgemeinheit bewiesen werden kann. Die Sache liegt demnach so: Im Herzmuskel der Amphibien fehlen bei reichster Golgiimprägnation freie Nervenenden ganz, folglich laufen die Endäste der Nervenfasern ineinander über. Je unvollständiger die Imprägnation, desto mehr scheinbare freie Enden treten auf. An den Radiärmuskeln der Chromatophoren lässt sich dieses schlingenförmige Ineinanderlaufen bei guter Methylenblaufärbung direkt erweisen, bei unvollständiger Farbung treten auch hier nur freie Endigungen in der Nähe der Chromatophore auf. Ich glaube keiner Opposition zu begegnen, wenn ich daraus den Analogieschluss ziehe, dass allgemein auch an den Chromatophoren die Nervenfasern schlingenförmig miteinander anastomosieren, und dass das Abbrechen der Färbung in der Nähe des Chromatophorenkörpers nur scheinbare freie Endigungen vortauscht.

Freilich wäre es möglich, dass auch diese weitestgehenden Färbungen, welche ich bisher erhalten habe, noch nicht ganz vollständig alle nervösen Elemente sichtbar machen. Es lässt sich nicht ausschliessen, dass vielleicht von den gefärbten Nervenendschlingen noch ungefärbte seitliche Ästchen abgehen, die ihrerseits an oder in der Substanz der Muskelzelle frei endigen Fin Beweis dafür ist allerdings an unseren Präparaten nicht zu erbringen. Die ganz blass gefärbten seitlichen Schleifen und Fadchen an den Stellen a und b der Fig. 4 auf Taf. XXI, welche von der Nervenschlinge ab über die Muskelsubstanz in der Nähe das Chromatophorenkörpers hin verfolgt werden können, sind wohl nichts anderes als Schlingenbildungen der einzelnen Fädchen des Nervenbündels, dessen Elemente hier auseinander weichen, und wenn eines davon frei $\mathrm{zu}$ endigen scheint, so kann das ganz wohl 
auf dem Aufhören der ohnehin sehr blassen Färbung beruhen. Man muss die Möglichkeit des Abgehens seitlicher Ästchen von den Nervenschlingen eigentlich bloss deswegen offen halten, weil an einem anderen Objekte das Eindringen solcher Stiftchen in die kontraktile Substanz hinein mehrfach angegeben worden ist, nämlich an glatten Muskelzellen des Blutegels und zwar speziell an der "mesenchymatösen" Muskulatur des Verdauungsstraktes und der Blutgefässe (Ranvier, ${ }^{1}$ ) Heymans ${ }^{2}$ ). Ich habe gar nicht die Absicht, hier weiter auf diese Frage einzugehen, weil ihre Beantwortung für unsere Fragestellung belanglos ist. Sollten nämlich wirklich von den Nervenschleifen der Radiärnerven noch weitere bis jetzt nicht nachgewiesene seitliche $Z$ weige mit freien Endigungen abgehen, so würde dies nichts an der Tatsache ändern, dass kurz vorher - präterminal - eine Vereinigung der Nervenfasern untereinander erfolgt. Man würde also höchstens statt des Wortes „Endplexus" den Ausdruck „präterminaler Plexus" $z u$ setzen haben.

Alles in allem sind wir nunmehr bei der Innervation der Chromatophoren genau bei derselben alternativen Fragestellung angelangt, die wir seinerzeit schon für das Herz aufgestellt haben : Wenn wirklich die terminalen (eventuell präterminalen) Nervenästchen ohne zu enden ineinander hinein verlaufen, bilden bloss die Endverzweigungen einer jeden Stammfaser untereinander Endschleifen, oder besteht ein durchgehendes (kontinuierliches) peripheres Nervennetz, hergestellt durch unbeschränkte Anastomosenbildung zwischen den Endverzweigungen aller Stammnervenfasern untereinander?

Ich habe, um diese Frage womöglich zu entscheiden, an verschiedenen anscheinend besonders günstigen. Stellen den Versuch gemacht, die an die Chromatophoren herantretenden Nervenfädchen von der Chromatophore weg gegen ihren Ursprung hin $\mathrm{zu}$ verfolgen. Das ist aber ganz ausserordentlich schwierig. Wir wissen schon, dass am Radiärmuskel entlang gewöhnlich ein ganzes Bündel von mehreren Einzelfädchen hinläuft. Diese Bündel gehen

1) Appareils nerv. terminaux des muscles de la vie organique. p. $493 \mathrm{ff} .1881$.

-) Exposé de l'état actuel de nos connaissances et observations personnelles sur la terminaison des nerfs dans les muscles lisses de la sangsue, précédés de la bibliographie des travaux parus sur linnervation des muscles lisses en général. Bruxelles. 1889. 
nun am distalen Ende der Radiärfaser über in das Gewirr des Endplexus, welches in Begleitung der Muskelzüge die Haut durchzieht. Darüber orientiert man sich im allgemeinen schon bei schwacher Vergrösserung. So erkennen wir in Fig. 2 auf Taf. XXI in den gegeneinander konvergierenden Fäden $a, b$ und $c$ unschwer die an drei benachbarten Radiärfasern einer nicht eingezeichneten Chromatophore zustrebenden Nervenbündel und sehen ihren Übergang in das unregelmässige Geflecht, welches das ganze Gesichtsfeld durchzieht. Wir sehen ferner (am Ende des Faserzuges a), dass sich das Nervenbündel distal teilt und dass jeder Teil in ein anderes Bündel des Hautmuskelplexus einmündet. Verfolgt man mit enger Blende und starker Vergrösserung die Nervenbündel genauer, so sieht man, dass ihren Teilungen auch Teilungen der Radiarmuskeln entsprechen, die ăhnlich, wie dies schon S te in ach in seiner oben S. 365 zitierten Abhandlung (Fig. 4 und 6 auf Taf. I) abbildet, an verschiedene Bündel von Hautmuskelfasern herantreten. An solchen Stellen muss man nun freilich die Hoffnung aufgeben, den Ursprung der einzelnen Fasern des Radiärnervenbündels festzustellen. Nur ganz ausnabmsweise findet man aber doch Stellen, wo die Nervenfädchen der Radiärmuskeln unmittelbar oder mit ganz kurzem Zwischenstück aus einer breiteren Nervenfaser des Grundplexus entspringen. Eine solche Stelle ist in Fig. 3 auf Taf. XXI abgebildet. Hier entspringen zwei benachbarte Nervenfädchen einer Chromatophore f und $\mathrm{g}$ allem Anschein nach aus einer und derselben Nervenfaser des Grundplexus. Leider ist in diesem Falle die Färbung der Schleife am Chromatophorenkörper nicht vollständig, sodass die Annahme, dass hier eine Anastomose zwischen zwei Endästen einer Nervenfaser eine wenn auch sehr wahrscheinliche, aber doch nicht endgültig bewiesene Vermutung darstellt. Ganz äbnlich liegen die Verhältnisse an einer anderen, nicht abgebildeten Stelle.

Das Resultat ist demnach in dieser Hinsicht recht mager. Im ganzen kann man sagen, dass für die schleifenförmige Verbindung der Endverzweigungen ein er Stammfaser untereinander wenigstens Stellen anzuführen wären, an welchen der geschlossene Nachweis wegen der mangelbaften Färbung zwar nicht erbracht, aber nach der ganzen Konfiguration mit grosser Wahrscheinlichkeit zu erschliessen ist. Für die Annahme eines völlig kontinuierlichen Nervennetzes, das durch Anastomosen der Endverzweigungen 
aller Stammnervenfasern untereinander gebildet würde, liess sich rein histologisch weder pro noch contra ein direkter Beweis erbringen. Für diese Annahme liessen sich vom histologischen Standpunkte aus höchstens Überlegungen ganz allgemeiner Natur anführen. Man muss sich nämlich daran erinnern, dass ja im Zentralnervensystem die an einer Ganglienzelle endigenden Endkörbe verschiedener Herkunft in der Tat ein gemeinsames Nervenendnetz bilden und kann daraus die Vermutung ableiten, dass analoge Verhältnisse auch an peripheren Nervenendigungen vorliegen mögen. ${ }^{1}$ ) Ausschlaggebend sind natürlich solche Überlegungen keineswegs. Eher könnte man noch eine Entscheidung von der Erhebung des physiologischen Tatbestandes erwarten. Das physiologische Experiment wird wenigstens das eine lehren, ob eine kontinuierliche Erregungsleitung in der Peripherie besteht oder nicht. Ich will hier gleich vorwegnehmen, dass eine solche $\mathrm{nicht}$ nachweisbar ist. Die weitere Diskussion dieses Ergebnisses kann freilich erst im physiologischen Teil dieser Untersuchungsreihe erfolgen, der in Pflügers Archiv veröffentlicht wird.

\section{Innervation der Flossenmuskulatur bei Cephalopoden.}

I)ie Innervation der Flossenmuskulatur habe ich besonders bei den kleinen Arten (Sepiola Rondeleti, Sepia elegans) gut studieren können, doch besitze ich auch Präparate von Loligo vulgaris und Sepia officinalis. Leider liefert die Methylenblaumethode bei diesen Objekten keine so vollständigen Färbungen, wie an den Chromatophoren, sodass es mir bloss möglich ist, die allgemeinen Grundzüge der Innervation, wie sie sich nach meinen Präparaten darstellen, anzugeben. Diese sind nun den bereits ausführlich geschilderten Verhältnissen an den Chromatophoren ausserordentlich ähnlich. Auch hier bilden die dickeren Nervenfasern, welche in den vom Zentralnervensystem herkommenden Nervenästen enthalten sind, zunächst mit ihren Verzweigungen einen weit ausgedehnten Grundplexus, den ich allerdings bloss in der Flosse der kleineren Tiere (Sepiola) beobachten konnte, wo seine Bündel nur aus wenigen Nervenfasern zusammengesetzt

1) Darauf sowie auf die Angaben von Apàthy über das Verhalten der Neurofibrillen in der Peripherie komme ich in den physiologischen Abhandlungen in Pflügers Archiv ausführlicher zu sprechen. 
sind. Von diesem Grundplexus gehen dann feine Ästchen zur Muskulatur ab. Letztere stellt sich in der Aufsicht auf die Flosse dar als aus schon makroskopisch sichtbaren Längsbündeln bestehend, die sich unter dem Mikroskop als Bündel dicht aneinander geschmiegter Spindelzellen erweisen, welche sich häufig mit $\mathrm{Me}-$ thylenblau sehr schön färben. Diese Längs-, richtiger Radiärbündel, welche in der Aufsicht geradeso aussehen, wie die Muskelbündel des Amphibienherzens, erstrecken sich, wie man an Querschnitten sieht, in die Tiefe der Flosse, sind also eigentlich Längsschichten. Sie anastomosieren vielfach miteinander in der Weise, dass sich eine Schicht von Spindelzellen von dem einen Zug abspaltet und schräg zum benachbarten hinüberzieht (man vergl. den Zug bei $\mathrm{C}$ in Fig. 7 auf Taf. XXI). Zwischen den Längsschichten der Muskulatur sieht man ein in der Aufsicht an Methylenblaupräparaten eine feine, blasse Netzzeichnung aufweisendes Gewebe, welches an Querschnitten, die nach van Gieson gefärbt sind, als eine Schicht von die Dicke der Flosse durchsetzenden Muskelbündeln verifiziert werden kann. ${ }^{1}$ ) Nach aussen von dieser derben, einheitlichen Muskelmasse, die demnach aus regelmässig abwechselnden Zügen von Längs- (Radiär-) und Transversalmuskeln besteht (im Inneren der Flosse finden sich noch sagittale Muskelzüge), setzen an der Flosse Muskelzüge an, welche vom Rücken her divergierend über die Flosse hin ausstrahlen, zu den tieferen Schichten der Hautmuskulatur gehören und nach der Art ihres Ansatzes vermutlich als Flossenheber funktionieren. Die Elemente dieses Muskels sind sehr häufig büschel- oder sternförmig weitverzweigt und ihre mit Methylenblau gefärbten Enden könnten sehr leicht mit Nervenfasern, oder gar die ganzen Zellen mit Ganglienzellen verwechselt werden.

Bei Loligo und Sepia treten die Nerven, soweit ich mich darüber orientieren konnte, in die Mittelschichten der Flossenmuskulatur ein, wenigstens gelangen die letzten Zweige von innen heraus zu den oberflächlichsten Muskelschichten. Diese Nervenfasern treten dann in das Innere der Muskelschichten ein und bilden zwischen den Elementen derselben einen sehr stark vari-

1) Ich bemerke ganz ausdrücklich, dass es hier nicht meine Absicht ist, auf die Details in der Anordnung der Flossenmuskulatur einzugehen. Ich beabsichtige nur, das zur Erklärung der Innervation Notwendige von jenen Stellen, wo ich die Nerven färben konnte, zu erwähnen. 
kösen Endplexus. Leider ist es ganz ausserordentlich schwer, das Bild dieses Endplexus auf einer Zeichnung wiederzugeben, weil die Verzweigung stark auch nach der Tiefe erfolgt und man dann auf der Zeichnung die tiefen und oberflächlichen Fädchen des Endplexus auf einer Fläche zusammendrängen muss, was zu falschen Vorstellungen führen kann. Unter einer gewissen Reserve verweise ich daher auf den Versuch, in Fig. 7 auf Taf. XXI diesen Endplexus von zwei benachbarten Längsschichten (und teilweise von der dazwischen liegenden Transversalschicht) wiederzugeben. Man sieht hier, wie eine Nervenfaser a sich teilt, um in den Endplexus zweier benachbarter Längsschichten A und B einzugehen, dass ferner ein und dieselbe Längsschicht B von zwei verschiedenen Nervenfasern $a^{\prime \prime}$ und $b$ mit Nervenendgeflechten versorgt wird. Schlecht zu sehen ist aus dem oben angeführten Grunde die am Präparate selbst leicht zu konstatierende Tutsache, dass die varikösen Fädchen des Endplexus innerhalb der Längsschichten der Muskulatur darin bleiben und in derselben Weise, wie es beim Herzmuskel der Amphibien der Fall ist, zwischen den Muskelzellen hinziehen. Wo ein scheinbares Heraustreten der Fasern des Endplexus aus der Muskelschicht erfolgt, wie z. B. an der mit $\mathrm{D}$ bezeichneten Stelle der Figur, dort bemerkt man mit enger Blende, dass auch ein abzweigender Muskelzug vorbanden ist. Die Faser c in derselben Figur, welche im Transversalmuskel oberhalb des tiefer liegenden Längszuges $D$ verläuft, steht mit den Fasern $a^{\prime}$ und $a^{\prime \prime}$ in keinem Zusammenhang.

Vergleichen wir den hier gefärbten Endplexus mit dem in den Herzmuskelbündeln der Amphibien befindlichen ganz analogen „intramuskulären" Plexus, so wird man sich sagen müssen, dass offenbar in unserem Falle nur eine ganz unvollständige Fürbung vorliegt. Ganz so, wie z. B. an den Chromatophoren die Färbung in der Nähe des Pigmentkörpers nur selten gut gelingt, so wird auch in der Flossenmuskulatur die Färbung immer schlechter, je weiter man sich vom Grundplexus entfernt und zwar wiederholen sich dann alle jene Bilder, die ich seinerzeit beim Herzmuskel schon genau beschrieben und kritisiert habe. $\left.{ }^{1}\right)$ Ich kann demnach auch die scheinbaren freien Nervenenden, die hier sichtbar werden, nicht als wirkliche Endigungen an den Muskelzellen, sondern bloss als durch Aussetzen der Färbung bedingte Kunstprodukte

1) His' Arch. 1902, S. 104 und 102. 
gelten lassen und bin überzeugt, dass eine Methode, welche eine vollkommene Nervenfárbung gestatten würde, auch innerhalb der Flossenmuskulatur dieselben durchgehenden, nirgends endenden Nervenschleifen ergeben würde, wie wir sie von der Chromatophorenmuskulatur ber kennen und beim Herzmuskel aus dem Fehlen freier Enden bei reichster Imprägnation erschlossen haben. Darnach würde sich auch für die Flossenmuskulatur die oben gestellte Frage erheben, ob es ein kontinuierlich geschlossenes Nervenendnetz innerhalb der Muskelschichten gibt, oder ob bloss Schleifenbildung zwischen den Endverzweigungen jeder einzelnen Stammnervenfaser unter sich anzunehmen sei.

Ich füge schliesslich noch hinzu, dass ich auch von der Mantelmuskulatur einiger Cephalopodenarten (Sepia officinalis und elegans) ganz ännliche Nervenbilder bekommen habe, wie von der Flossenmuskulatur. Allerdings war die Färbung hier ebensowenig vollstandig wie an der Flossenmuskulatur, und es ist auch nicht gelungen, die Präparate gut zu fixieren.

\section{Bemerkungen über die Innervation der glatten Muskulatur der Wirbeltiere.}

Über die Aufteilung der Nerven innerhalb der glatten Muskulatur der Wirbeltiere (zu der auch noch die ibr verwandte Herzmuskulatur mindestens der Amphibien hinzuzufïgen wäre) herrscht im ganzen eine sehr erfreuliche Übereinstimmung.') Von Anfang an wurde von den Autoren unterschieden ein grösstenteils aus stärkeren Nervenfasern zusammengesetzter Grundplexus, welcher die Anordnung der Muskelbündel nicht einhält, welcher sich dann immer mehr und mehr aufteilt, sodass schliesslich an die Muskelbündel selbst nur noch einzelne oder in geringer Zahl vereinigte Nervenfasern herantreten. Gewöhnlich wird dieser Übergangsteil als eigener intermediärer (Klebs, Löwit) oder

1) Ich glaube wohl von einer ausführlichen Literaturzusammenstellung um so eher absehen zu dürfen, als solche schon mehrfach vorliegen. So für das gesamte Gebiet bis zum Jahre 1888 in der oben S. 380 zitierten Abhandlung von Heymans; über die Nerven des Verdaunngstraktes, sowie über die glatte Muskulatur überhaupt bei E. Müller (Zur Kenntnis der Ausbreitung und Endigungsweise der Magen-, Darm- und Pankreasnerven, Arch. f. mikr. Anat., Bd. 40, S. 390, 1892); über die Gefässnerven bei La a insky Über die Gefässinnervation der Hundepfote, Arch. f. mikr. Anat., Bd. 65, (S. 623, 1905). 
perimuskulärer (Gerlach) Plexus besonders unterschieden. Von ihm erst gehen dann die ganz feinen Fadchen ab, welche in die Muskelbündel selbst eindringen, zwischen den Muskelzellen und an ihnen entlang hinziehen, wobei sie sich allerdings gabeln, auf kurze Strecken auch quer verlaufen können, im ganzen aber immer sehr genau die Anordnung der Muskelzellen innerhalb des Bündels wiedergeben, sodass man diese daraus direkt diagnostizieren kann, wie dies insbesondere in den Arbeiten aus dem Arnsteinschen Laboratorium betont wird. Diese alle glatten Muskeln in überaus reicher Zahl durchflechtenden Nervenfädchen bilden den intramuskulären Plexus, den ich oben S. 373 als Endplexus bezeichnet habe.

Ich bin ebenso wie $0 \mathrm{penchow} \mathrm{si}^{1}$ ) der Meinung, dass man mit der Unterscheidung zwischen Grundplexus und Endplexus auskommt und dass die Abtrennung eines besonderen intermediären oder perimuskulären Übergangsplexus unterbleiben kann. Die Trennung von Grundplexus und Endplexus halte ich aber Heymans und Demoor ${ }^{2}$ ) gegenüber, welche der Unterscheidung zwischen einem "plexus on plus exactement, réseau périfasciculaire et intrafasciculaire, fondamental et périphérique " eine scharfe anatomische Charakterisierung aberkennen, für unbedingt nötig.

Während nun diese Art der Aufteilung der Nerven innerhalb der glatten und der ihr verwandten Muskulatur allgemein zugegeben wird, so gehen die Meinungen vollkommen auseinander darüber, ob die Nervenfasern innerhalb der Muskulatur ein zusammenhängendes Netz bilden, oder ob die einzelnen Fadchen frei an oder in den Muskelzellen endigen. Vergegenwartigen wir uns nun, mit welchen Methoden man denn überhaupt diesen intramuskulären Endplexus dargestellt hat, so sehen wir, dass es lauter „unzuverlässige" Methoden sind, die selten alles Nervöse, in der Regel nur einen Teil davon färben (Gold-, Methylenblau-, Golgimethode). Nun ist es ganz klar, dass unter diesen Umständen jene Präparate die entscheidenden sein werden, in welchen die Farbung am weitesten gegangen ist. Wenn demnach bei Gold-, Methylenblau- oder Golgipräparaten die einen Autoren die Fädchen des intramuskulären Plexus frei endigen sehen, andere sie aber immer weiter und weiter von einer Muskelzelle zur

1) Beitrag zur Kenntnis der Nervenendigungen im Herzen. Arch. f. mikr. Anat., Bd. 22, S. 415 ff., 1883.

2) Etude sur l'innervation du coeur des vertébrés à l'aide de la méthode de Golgi. Arch. de Biol., T. 13, pag. 645, 1894. 
anderen verfolgen können und keine Endigung finden, so sollte man doch wohl meinen, dass eben im letzteren Falle als der weitergehenden Färbung der richtige Sachverhalt zum Vorschein gekommen ist. Wie spärlich die Färbung bei vielen Autoren gewesen ist, geht daraus hervor, dass die Frage aufgeworfen wurde, ob denn jede einzelne Muskelzelle von einer Nervenfaser versorgt wird, ja dass sie sogar gelegentlich verneint wurde! Wer gute Nervenfärbungen in der glatten Muskulatur gesehen hat, wird dem nicht beipflichten können. Nicht, ob jede Muskelzelle von einer, nein, ob sie von mehreren Nervenfasern versorgt wird, sollte man fragen. Meiner Meinung nach sind Untersuchungen, in welchen die Versorgung jeder einzelnen glatten Muskelzelle durch einen Nerven bezweifelt wird, als solche mit unzureichender Nervendarstellung für unsere Frage ganz auszuscheiden, und auch von den übrigen Untersuchungen sind nur solche in Betracht $\mathrm{zu}$ ziehen, bei welchen die Färbung am weitesten geht. Dort aber, wo sie am besten gelungen ist, siebt man auch keine freien Nervenenden mehr! „Was endlich freie Nervenendigungen zwischen den Muskelzellen betrifft, "sagt Lö wit') bezüglich der Muskulatur der Harnblase, „so ist eine definitive Entscheidung darüber nach Goldfärbungen allerdings nicht möglich, da ja noch immer Nervenfasern durch das Gold ungefärbt geblieben sein konnten. Ich hebe aber hervor, dass ich an meinen gelungensten Goldpräparaten immer nur zusammenhängend e ${ }^{2}$ ) Endfibrillen gesehen habe."

Ähnlich spricht sich Gonjae ${ }^{3}$ ) aus über die Nervenfädchen, welche zwischen den Muskelzellen der Gefässe verlaufen: „An gel ung en en ${ }^{4}$ ) Präparaten sieht man keine freien Endigungen, auch hier haben wir es mit einem Terminalnetz zu tun." Das ist doch genau dasselbe, was ich oben von der Innervation der Chromatophoren sagen konnte. An den ge lung ens te n Präparatstellen sieht man keine freie Endigung, sondern schleifenförmiges Ineinanderlaufen der Nervenfasern. Ich habe mir nun in Innsbruck neuerdings eine grössere Zahl von Methylenblaupräparaten

1) Die Nerven der glatten Muskulatur. Sitzgsber. d. Wiener Akad. d. Wiss., Bd. 71, Abt. 3, S. 376, 1875.

2) Von mir gesperrt gedruckt.

3) Die Nerven des Nahrungsschlauches. Arch. f. mikr. Anat., Bd. 11, S. 489,1875 .

4) Von mir gesperrt gedruckt. 
aus dem Darmtrakt und der Blase des Frosches bergestellt und kann mich nach den Bildern, die ich gesehen habe, auch bezüglich der glatten Muskulatur der Wirbeltiere nur der Meinung jener Autoren anschliessen, welche annehmen, dass die intramuskulären Nervenfädchen ein wirkliches ineinander laufendes Netz bilden, wieder aber mit der mehrfach schon betonten noch zu entscheidenden alternativen Fragestellung, ob dies Netz ein kontinuierliches oder für jede Stammnervenfaser gesondertes ist. $\mathrm{Ob}$ freilich, um auch dies nochmals zu betonen, von diesem intramuskulären Netz noch kurze Seitenzweigchen in die Muskelzellen oder ihre Kerne hinein abgehen, wie dies von mehreren Seiten behauptet worden ist, lasse ich bis auf weiteres dahingestellt und verweise nur noch auf meine Bemerkungen über den Zusammenhang zwischen Nerv und Muskel auf S. 106 meiner oben, S. 362 zitierten Abhandlung.

Nur über den Herzmuskel der Säugetiere möchte ich bezüglich del hier besprochenen Frage noch Zweifel übrig lassen. Zwar will E. Fischer ${ }^{1}$ ) mit der Goldmethode ein zusammenhängendes intramuskulares Nervennetz dicker bis feinster Fasern dargestellt haben, deren freie Enden, wenn sie vorkommen, wie abgerissen aussehen, ohne besondere, eine Endigung kennzeichnende Bildung, die er demnach nicht als wirkliche Endigungen, sondern als Aufhören der Färbung betrachtet. Dagegen hat Jacques²) mit der Golgimethode allerdings besondere knöpfchenförmige oder mehrzipfelige Endigurgen dargestellt, freilich nicht bloss am Ende der intramuskulären Nerven, sondern auch in ihrem Verlauf. Es würde sich also dann doch noch fragen, ob diese Bildungen nicht bloss besonders grosse Varikositäten waren, jedenfalls ist die Frage noch nicht endgültig $z u$ entscheiden.

\section{Über die Betheschen „Nervennetze“.}

Wenn innerbalb der glatten Muskulatur (und der ihr verwandten Herzmuskulatur) eine netzförmige Anastomosenbildung zwischen Nervenfasern verschiedener Herkunft vorhanden sein sollte, so müsste sie nach den bisherigen Darlegungen im End-

1) Über die Endigung der Nerven im quergestreiften Muskel der Wirbeltiere. Dies Arch., Bd. 13, S. 38 ff., 1877.

2) Recherches sur les nerfs du coeur chez la grenoville et les mammi-

fères. Journ. de l'anat. et de la physiol., 1894, S. 622. 
plexus erfolgen. Innerhalb der Geflechte des Grundplexus sind Queranastomosen zwischen den nebeneinander liegenden Nervenfasern nirgends nachzuweisen. Gesetzt nun, es existiere ein solches kontinuierliches peripheres Endnetz, in welcher Beziehung würde es stehen zu den von Bethe angenommenen Nervennetzen? Unter Nervennetzen versteht Bethe (Allg. Anat. u. Physiol. d. Nervensystems, S. 78 ff.) eine besondere Form des Nervensystems, welche charakterisiert sein soll durch breite Anastomosen zwischen den (Ganglien-) Zellen. Eine Unterscheidung der Zellfortsătze „in Protoplasmafortsătze und Achsenzylinderfortsätze ist unmöglich. Nach meist kurzem Verlauf, während dessen spärliche Seitenzweige abgegeben werden, vereinigen sich die Fortsätze wieder mit anderen Zellen, meist schon mit der zunächst gelegenen." Solche Nervennetze sollen nun vorkommen, ausser an einer Anzahl von anderen Stellen, von denen ich hier absehe, insbesondere auch bei den Mollusken und im Blutgefässsystem der Vertebraten. Nur mit dieser Angabe will ich mich hier beschäftigen. Am deutlichsten geht die Art, wie sich Bethe die peripheren Nervennetze vorstellt, hervor aus einem Schema, das er in der Deutschen med. Wochenschr. ${ }^{1}$ ) veröffentlicht hat und das als das Wesentlichste die dicken Anastomosen zwischen den Ganglienzellen zeigt, welche miteinander das "Nervennetz" bilden, während die rom „Nervennetz ${ }^{*}$ zur Muskulatur abgehenden dünnen Nervenfäserchen nur angedeutet sind.

Vergleicht man die Darstellung Bethes und sein Schema mit unseren früheren Ausführungen, so sieht man sofort den fundamentalen Unterschied zwischen beiden. Bethes „Nerven-

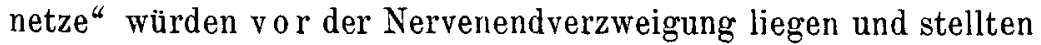
sich dar als Ganglienzellanastomosen. Erst von ihnen weg gingen dann die sich verzweigenden (und frei endigenden?) Seitenästchen zur Muskulatur ab. Nach unserer Darstellung bilden aber gerade diese zu den Muskelzellen hinziehenden Ästchen untereinander das periphere Netz, wenn ein solches überhaupt existiert. Ein Nervennetz mit Ganglienzellen im Sinne von Bethe aber vor den Endastchen zur Muskulatur ist mit der Methylenblaumethode weder an der Innervation der Chromatophoren, noch an der Innervation der

1) Der heutige Stand der Neuronentheorie. Deutsche med. Wochenschr. 1904 , S. $1 \geq 02$. 
Flossen-und der Mantelmuskulatur der Cephalopoden, noch auch, nach meiner früheren Abhandlung, im Herzmuskel nachweisbar.

Durch diesen Satz gerate ich allerdings in Widerspruch zu Bethe, und es wird notwendig sein, zu untersuchen, wie Bethe zu seiner Annahme von den oben beschriebenen Nervennetzen in der Herz- und Gefässmuskulatur der Wirbeltiere gekommen sein kann. Vorausschicken möchte ich, dass es sich bei dieser Frage stets nur um die spezifischen „B e the schen Nervennetze" handelt, die einen ganz charakteristischen Bau aufweisen sollen, der von dem des übrigen Nervensystems abweichen soll, also keineswegs etwa um die Frage nach dem Zusammenhange jener gangliösen Geflechte mit der Muskulatur, wie sie z. B. in sympathischen Geflechten, eventuell auch innerhalb der Organe (wie im Wirbeltierherzen und Darm) vorhanden sind, oder wie

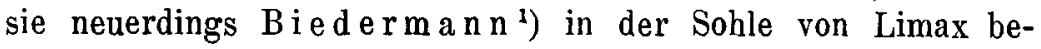
schrieben hat. Der Bau der sympathischen Ganglien der Wirbeltiere ist ja jetzt in seinen Grundzügen so ziemlich erkannt und stimmt nicht mit dem der Betheschen Nervennetze überein, sondern ordnet sich vielmehr dem Neuronentypus ein, insofern als man Nerven- und Protoplasmafortsatze auseinander halten und den Nervenfortsatz gewisser Ganglienzellen als „Stammnervenfaser" im oben S. 362 definierten Sinne bis zur Muskulatur hin verfolgen kann. Der feinere Bau der gangliösen Netze in der Sohle von Limax ist noch nicht untersucht worden, und es ist daher abzuwarten, ob sich hier eine den spezifischen Beth e schen Nervennetzen analoge Bildung wird nachweisen lassen.

Wir fragen nun, gibt es, zunächst bei der Muskulatur der Cephalopoden, irgendwelche, in der bisherigen Darstellung nicht besprochene Elemente, welche auf die Existenz spezifischer Bethescher Nervenetze hindeuten? Solche gibt es allerdings. $\mathrm{Chun}$, dem bei seinem durchsichtigen Objekt, an welchem sich die Formelemente des Nervensystems so scharf von der gallertigen Umgebung abheben, ols ob man es mit einem durchsichtigen

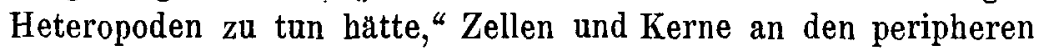
Nerven unmöglich entgehen konnten, bildet in der Tat an den die Chromatophoren versorgenden Nerven Kerne ab, welche teils

1) Studien zur vergleich. Physiologie der peristalt. Bewegungen. 3. Die Innervation der Schneckensohle. Pflïgers Arch., Bd. 111, S. 251, 1905. 
in den dickeren Nervenstämmchen liegen (Fig. 6 und 7 der Abhandlung von $(\mathrm{Chun}$ ), teils in den meist dreieckigen plattenförmigen Verbreiterungen an den T'eilungsstellen der feineren Nervenästchen. Insbesondere an den letzterwähnten Stellen kann nach $\mathrm{Chuns}$ Fig. 3 (S. 170 seiner Abhandlung) fast genau das Bethesche Schema auftreten, das wir oben erwähnt haben. Wie sind nun solche Stellen zu vereinbaren mit dem oben aufgestellten Satze, dass es bei der Innervation der Chromatophoren keine Betheschen Nervennetze gibt?

Kerne an marklosen Nerven von Mollusken sind schon oft beschrieben worden, so zuerst von Leydig, ${ }^{1}$ ) dann von $\mathrm{Boll}^{2}$ ) and Ranvier. ${ }^{3}$ ) Leydig hält die von ihm an sensiblen Hautnerven eines Heteropoden gefundenen Kerne mit Plasma für Ganglienzellen. Boll bestätigt die Beobachtung, spricht sich aber nicht weiter darüber aus. Dagegen erwähnt er, dass die stärkeren Nervenfasern in der Haut der Cephalopoden von einem echten, aus platten Bindegewebszellen zusammengesetzten Neurilemm bekleidet sind, „auf dessen Existenz die der Nervenfaser anliegenden ovalen Kerne hinweisen". Ranvier beschreibt an den Nervenfasern der Schnecke Kerne einer Nervenscheide, die er der Henle schen Scheide der Wirbeltiernerven homolog setzt. Diese Scheide und ihre Kerne habe ich bei Loligo insbesondere mehrfach an einem Nervenplexus (Grundplexus) mit Methylenblau gefärbt gefunden, welcher der Flosse nach dem Abziehen der Haut aufliegen bleibt und dessen Endplexus allem Anschein nach nicht in der eigentlichen Binnenmuskulatur der Flosse sich befindet, sondern in den unmittelbar darüber liegenden Partien der Hautmuskulatur. Hier ist nun an besonders günstigen Stellen (vergl. Fig. 8 auf Taf. XXI) die Nervenscheide daran kenntlich, dass sich auf ihr ein körneliger, blass gefärbter Niederschlag abgesetzt hat..) Die Scheide ist an der abgebildeten Stelle von der Nervenfaser,

1) Anatom. Bemerkungen über Carinaria, Firola und Amphicora. Zeitschrift f. wiss. Zool., Bd. 3, S. 325, 1851.

") Beiträge zur vergl. Anatomie des Molluskentypus. Dies Arch., Bd. 5, Suppl. S. 20, 1869. Hier sind noch Gegen ba ur und Le ukart zitiert.

3) Appareils nerv. terminaux des muscles de la vie organique. Paris 1881, S. 991.

t) Auf der Zeichnung ist bloss der optische Querschnitt dargestellt. Bei hoher Einstellung kann man die Körnelung über die ganze Nervenfaser hinweg verfolgen. 
welche innerhalb derselben in schwachen Krümmungen verläuft, abgehoben. Der Kern liegt der Scheide an ihrer Innenfläche an und ist deutlich von der Nervenfaser getrennt. Dass die Scheide auch auf den seitlich abgehenden Nervenast übergeht, sieht man an der körneligen Umrandung jener Stelle, wo die Nervenfaser die Scheide nicht ganz ausfüllt. Dort, wo sich kein Zwischenraum zwischen Scheide und Nervenfaser infolge der Behandlung des Präparates gebildet hat, kann man die Scheide auch bei starken Vergrösserungen nicht als gesonderten Rand von der Nervenfaser trennen. Sind in einem solchen Falle die Kerne der Scheide mitgefärbt, so liegen sie dem Nerven unmittelbar an und zwar so dicht, ${ }^{1}$ ) dass sie wie in die Substanz des Nerven eingebettet erscheinen, sodass man ohne genauere Kenntnis der Verhaltnisse sehr leicht auf die Vermutung kommen könnte, die Nervenfaser sei mit den Kernen zu einem Zellganzen verschmolzen. Dass dies nicht der Fall ist, geht aber ausser aus dem Vergleich mit Stellen, wo die Scheidenkerne sich von den Nervenfasern sondern lassen, auch daraus mit Sicherheit hervor, dass sich die Kerne mit der ihnen anliegenden Nervenfaser nur sehr selten mitfärben, vielmehr ganz gewöhnlich die Nervenfasern sehr schön gefärbt sind, von den Kernen aber kein einziger. Dies ist in meinen Präparaten wohl ausnahmslos der Fall bei den Nerven der Chromatophoren.

Nun könnte man die Tatsache, dass sich zwar die Nervenfasern, aber nicht die ihnen anliegenden Kerne gefärbt haben, vielleicht auch so erklären wollen, dass nur die Neurofibrillen gefärbt seien, nicht aber das Neuroplasma mit seinen Kernen. Das geht aber nicht an, denn es wurde schon oben S. 371 darauf hingewiesen, dass in meinen Präparaten die Neurofibrillen zwar an manchen Stellen als feine, wellige Langsstreifung etwas hervortreten, im übrigen aber Fibrillen und Interfibrillarmasse zusammen gefärbt sind. Die Existenz von den Nervenfasern abgesonderter Scheidenkerne ist demnach jedenfalls zuzugeben, sie müssen an genügend durchscheinenden Objekten, wie z. B. in den Chunschen Präparaten, deutlich sichtbar sein und es besteht also

1) Hüten muss man sich nur davor, manche plötzlich ganz dunkel gefärbte Stellen der Nervenfasern, wie z. B. die Abgangsstelle des seitlichen Astes bei $\mathrm{c}$ in Fig. 8 als Kerne zu deuten. Nitunter kommen auch Knickungen der Nervenfaser vor wie an Stelle d der Fig. 8, die sich auch als dunkleres Querband kenntlich machen. 
zunächst die Möglichkeit, dass die von $\mathrm{Ch}$ un abgebildeten Kerne solchen Scheidenkernen einzelner Nerven fas er $n$ entsprechen. Es ist aber ausserdem noch daran zu denken, dass es auch Kerne eines die Nerven bü nd el einhüllenden Bindegewebes sein könnten. lch glaube übrigens nicht, dass jemand bloss aus der Existenz von Kernen in den gröberen Nervenstämmchen, die man ja in den dickeren zur Haut hinziehenden Nerven an Quer- und Längsschnitten auch färberisch reichlich nachweisen kann, schon auf die Anwesenheit von Ganglienzellen schliessen wird. Eher könnte man daran zweifeln, ob denn auch die Kerne an den eckigen Teilungsstellen der feinsten Nervenästchen in den Präparaten von $\mathrm{Chun}$ als Kerne von Nervenhüllen aufzufassen sind. Indessen wird man sich bei nahberer Überlegung doch kaum anders entscheiden können. Dass man diese Kerne an nicht spezifisch gefärbten Präparaten mit den Nervenbündeln in e in s verschmolzen sieht, bildet gar keinen Grund zu der Annahme, dass dies auch in Wirklichkeit der Fall ist. Man kann ja an solchen Präparaten auch nicht die einzelnen Nervenfaddchen, aus welchen die Nervenbündel zusammengesetzt sind, voneinander sondern. ${ }^{1}$ ) Das gelingt erst durch eine spezifische Nervenfärbung, wie die vitale Methylenblaubehandlung. An Methylenblaupräparaten aber lassen sich, wie oben ausfübrlich dargetan. worden ist, die Nerven der Chromatophoren von ihrem Ursprung aus den ganz breiten Nervenfasern des Grundplexus bis an den Chromatophorenkörper heran kontinuierlich verfolgen, ohne dass man Ganglienzellen eingeschaltet findet. Es müssten demnach diese Kerne in den eckjgen Teilungsstellen, wenn sie wirklich Kerne von Ganglienzellen sein sollten, zu einem gesonderten, von der Innervation der Chromatophoren von Seite des Zentralnervensystems unabhängigen Gangliensystem gehören, welches sich in meinen Präparaten trotz reichlichster sonstiger Nervenfärbung nicht mitgefärbt hätte. Das letztere wäre nun an sich nicht geradezu unmöglich. Es kommt bei Wirbeltieren vor, dass sich entweder die Ganglienzellen eines Organes überhaupt, oder wenigstens bestimmte Arten von Ganglien-

1) Aus diesem Grunde ist es auch unmöglich, in Präparaten ohne spezifische Nervenfärbung zu entscheiden, ob ein Nervennetz oder ein blosses Geflecht vorhanden ist, welche Schwierigkeit an einem anderen Objekt schon durch E. Cyon (Arb. a. d. physiolog. Anstalt z. Leipzig, 1869, S. 106 ff.) ausführlich erörtert worden ist. 
zellen mit Methylenblau sehr schwer färben. Aber in unserem Falle ist es doch sehr wenig wahrscheinlich, dass neben der reichen dargestellten zentrogenen Nervenversorgung noch eine gesonderte durch periphere Ganglienzellen vorhanden sein sollte. Jedenfalls wäre die Existenz eines solchen gesonderten Gangliensystems erst noch $\mathrm{zu}$ beweisen.

Aber auch aus Analogiegründen erscheint die Deutung der Kerne an den Teilungsstellen als Kerne von Ganglienzellen unwahrscheinlich. Es handelt sich bei diesen Stellen um die letzten feinsten Verteilungen des Grundplexus vor dem Abgange der terminalen Nervenasstchen zum Muskel. Wir werden im folgenden sehen, dass auch im Herzen und an den Gefässen der Wirbeltiere Kerne an den Teilungsstellen der letzten Verzweigungen des Grundplexus bäufig vorhanden sind. Diese Stellen können ganz ähnlich aussehen, wie jene in der Haut von Kephalopoden (man vergl. die Fig. 3 von $\mathrm{Chun}$ z. B. mit den Fig. 6 and 7 in der Abhandlung von Gerlach ïber die Nerven des Froschherzens ${ }^{1}$ ). Es ist also zunächst schon vorauszusehen, dass die Frage für alle diese Objekte in gleicher Weise zu beantworten sein wird, und wir werden sogleich sehen, dass sich auch im Herzen und an den Gefässen der Wirbeltiere die Deutung dieser Kerne als Kerne von Nervenhüllen ungezwungen ergibt.

Aber sind nicht wenigstens einige dieser Kerne Kerne von Ganglienzellen? Es könnte doch auch so sein, dass neben den Kernen der Nervenhüllen echte Ganglienzellen, aus welchen Chromatophorennerven entspringen, noch bis weit in die Peripherie hinaus zerstreut vorhanden sind. Darauf ist zu sagen, dass nach meinen Präparaten solche echte Ganglienzellen in der Rückenund Flossenbaut der von mir untersuchten Cephalopoden zu den grössten Seltenheiten gehören, aber doch ausnahmsweise mitunter vorkommen. ${ }^{2}$ ) Ganz sicher habe ich in einem Prapparate von Loligo zugleich mit der gewöhnlichen Färbung der Nervenfasern auch zwei Ganglienzellen mit Methylenblau gefărbt erhalten. Leider hat sich das Präparat beim Versuche der Fixation völlig entfärbt, sodass ich es bloss aus der Erinnerung erwähnen, aber

1) Virchows Arch., B. 66, Taf. 11, 1876.

2) Steinach gibt an (Pflügers Arch., Bd. 87, S. 20, 1901), dass er in der Chromatophorenschicht der Haut der Fangarme keine Ganglienzellen gefunden habe. 
keine weiteren Details geben kann. Die Tatsache aber, dass zwei unzweifelhafte Ganglienzellen von typischem Aussehen unipolarer Zellen sich so schön und leicht gefärbt hatten, war deshalb sehr wichtig, weil, wie oben bemerkt wurde, bei manchen Wirbeltierorganen die Ganglienzellen und ihre Fortsätze sich sehr schwer mit Methylenblau färben lassen. Das ist aber bei den echten Ganglienzellen in der Haut von Loligo offenbar nicht der Fall. Wenn demnach wirklich echte Ganglienzellen in grösserer Zahl vorhanden wären, müsste ich sie bei der ausserordentlich reichlichen Nervenfärbung vieler Präparate ebenfalls gefärbt finden. In Wirklichkeit befindet sich in meinen konservierten Präparaten bloss eine einzige Stelle, wo mit Wahrscheinlichkeit eine gefärbte unipolare Ganglienzelle samt Fortsatz anzunehmen ist. Es ist das die in Fig. 9 auf Taf. XXI abgebildete, in einem Nervenbündel liegende blau gefärbte Zelle, von welcher ein zunächst ziemlich breiter Fortsatz ausgeht, der allmählich schmäler wird, ganz so, wie man dies regelmässig an Golgipräparaten beim geraden Fortsatze der unipolaren Ganglienzellen des Froschberzens beobachtet. In der Zelle befindet sich an einer Stelle ein etwas hellerer Fleck mit einem dunklen Punkt, vermutlich der Kern mit dem Kernkörperchen. Auffällig ist nur die geringe Grösse der Zelle, welche sich mit $18 \times 6 \mu$ ungefähr in den Dimensionen der Scheidenkerne (durchschnittlich $20 \times 4 \mu$ ) hält. Man kann aber trotzdem nicht annehmen, dass die „Ganglienzelle“ etwa durch einen gut gefärbten Scheidenkern (die sich sonst an den Chromatophorennerven nie mit Methylenblau färben) vorgetäuscht wird, unter dem weg der scheinbare Fortsatz dann als gesonderte Nervenfaser hinziehen müsste und zwar so, dass seine Fürbung gerade unterhalb des Kernes aufhörte.

Für die Chromatophorenmuskulatur der Cephalopoden können wir demnach die Existenz peripherer, in die vom Zentralnervensystem herkommende Nervenversorgung eingeschalteter, gangliöser Nervennetze im Sinne von Bethe mit voller Sicherheit ausschliessen. Ja sogar das Vorkommen wirklicher Ganglienzellen ist in der Haut der Cephalopoden nur auf ganz vereinzelte Ausnahmen beschränkt. In der letzteren Beziehung steht es bekanntlich anders mit der glatten und der ihr verwandten Muskulatur der Wirbeltiere, zu deren Besprechung wir nun übergehen. Von jenen Stellen, an denen nach Bethe 
spezifische Nervennetze vorkommen sollen, ist es speziell das Herz, wo ausserdem wirkliche Ganglienzellen in grosser Zahl vorhanden sind. Aber diese Zellen mit ihrem wohlbekannten Typus echter Ganglienzellen unterscheiden sich durch ihre Form, Grösse und durch das Vorkommen von $\mathrm{Nis}$ i-Körpern wesentlich von den Betheschen "Ganglienzellen der Nervennetze", welche in der Regel sehr klein sind und keine $\mathrm{Nissl-Schollen}$ zeigen (Bet he, Allg. Anat. d. Nervensyst., S. 91). Es ist daher sachlich völlig begründet und wird für die weitere Diskussion sehr förderlich sein, wenn wir ganz ausdrücklich die unzweifelhaft echten Ganglienzellen, die teils zu Gruppen vereint, teils einzeln im Herzen vorkommen, einerseits und die sogenannten Ganglienzellen der Nervennetze andererseits scharf auseinanderhalten.

Die histologischen Verhältnisse der echten Ganglienzellen des Herzens sind im Săugetierherzen von A. S. Dogiel, $\left.{ }^{\prime}\right)$ im Froschberzen am ausführlichsten von $\mathrm{mir}^{2}$ ) untersucht worden, sie entsprechen den gewohnten Bildern sympathischer Ganglienzellen mit Nerven- und eventuellProtoplasmafortsätzen, interessieren uns also hier nicht weiter. Die Betheschen „Ganglienzellen der Nervennetze “ des Froschherzens dagegen, um die es sich hier handelt, sind offenbar identisch mit den im Herzen meines Wissens zuerst von L. Ge r l $\mathrm{ch}^{3}$ ) an Goldpräparaten beschriebenen Kernen des „perimuskulären“ Plexus, über welche dieser Autor folgendes sagt (1. c. S. 212): „Charakteristisch für sie (d. h. die Nervenfasern des perimuskulären Netzes) „sind die Kerneinlagerungen, welche vielfach sowohl im Verlaufe der Fasern, als in den Knotenpunkten des Netzes vorkommen; im ersteren Falle haben sie eine mehr spindelförmige Gestalt, im letzteren sind sie dreieckig*. Weiter (S. 213), nachdem er davon gesprochen hat, dass gewissen „Anhäufungen nervöser Substanz" an den Knotenpunkten des perimuskulären Netzes die Merkmale einer Zelle völlig abgehen, făhrt er fort: „Mit viel grösserem Rechte könnte man die kernhaltigen Knotenpunkte des Netzes als kleine Nervenzellen ansprechen, da sich bei ihnen, wenn auch nur in ausserst

1) Arch. f. mikr. Anat., Bd. อ̋3, S. 246, 1899.

2) His' Arch. 1902, S. 54.

$\left.{ }^{3}\right)$ Über die Nervenendigungen in der Muskulatur des Froschherzens (Virchows Arch., Bd. 66, S. 187; 1876). In der Harnblase beschrieb sie zuerst Klebs (Virchows Arch., Bd. 32, S. 168; 1865). Dort findet man auch die älteste Literatur über Kerne an solchen "Nervennetzen" überhaupt. 
seltenen Fällen, im Kern ein Kernkörperchen findet. Allein dieses inkonstante Vorkommen desselben, ferner der Umstand, dass die Hauptmasse der Zelle aus dem in seiner Form sehr wechselnden Kerne bestehen würde, muss zur Vorsicht mahnen, die kernhaltigen Knotenpunkte der perimuskulären Netze als wirkliche Zellen zu erklären." Illustriert werden diese Angaben von Gerl a $\mathrm{ch}$ durch die Fig. 6 und 7 seiner beigefügten Taf. XI, deren Betrachtung ich zum Verstăndnis des folgenden dringend empfehlen möchte.

Dass Bethe unter seinen Ganglienzellen des Nervennetzes wirklich diese Kerne des perimuskulären Plexus von Gerlach versteht, geht aus seiner Beschreibung derselben deutlich hervor (l. c. S. 93): „Die Ganglienzellen des Nervennetzes liegen nun meist an der 0 berfläch e ${ }^{1}$ ) der Muskeltrabekeln .... Sie haben zwei bis vier Fortsätze, welche sich in die Muskeltrabekeln hineinbegeben und sich dort in eine grosse Anzahl meist stark varikös gewordener Fasern aufsplittern." Das eigentliche Anastomosennetz liegt nach Bethe im Herzen teils in teils auf den Trabekeln, hauptsächlich aber im Innern der Muskeltrabekel (l. c. S. 93 und 94). Es weicht also die Verbindung der Ganglienzellen untereinander insofern von dem von Bethe in der Deutschen medizinischen Wochenschrift angegebenen Schema ab, als sie hauptsächlich intramuskulär erfolgt.

Utber diesen perimuskulären Plexus von Gerlach und die in ihm enthaltenen Kerne habe ich nun in meiner Abhandlung ( $\mathrm{H}$ i s' Arch. 1902, S. 95) folgendes gesagt: "Was Gerlach den perimuskulären Plexus nannte, waren, wie aus seinen Abbildungen und seiner ganzen Beschreibung hervorgeht, die letzten, aus nur wenig Nervenfasern bestehenden $Z$ weige des Grundplexus, die durch spärliche mitgefärbte Teile des intramuskulären Plexus netzartig miteinander verbunden waren. Da bei Gerlachs Metbode die Grenzen zwischen den einzelnen Nervenfädchen offenbar etwas verwischt blieben, so kam dadurch der Eindruck eines echten Netzwerkes zustande. Die von ihm erwähnten dreieckigen Kerne an den Teilungsstellen kann man auch an Methylenblaupräparaten nicht selten sehen, man sieht dann aber auch, $\left.{ }^{2}\right)$ dass sie an Nerven-

1) Von mir gesperrt.

2) Heute würde ich hier hinzufügen: „an guten Methylenblaupräparaten" (vergl. das Folgende). 
bündelchen liegen, deren Fasern alle an ihnen vorüberziehen Es sind diese Zellen also, was hervorzuheben nicht überflüssig sein dürfte, keine Ganglienzellen, die mit Nervenfasern in Verbindung stehen, sondern es sind offenbar die Zellen des die Nervenfasern begleitenden und einhüllenden Bindegewebes, das bei den etwas gröberen Nervenstämmchen eine deutliche Scheide bildet." Ich möchte gleich hier einschalten, dass bereits in der Abbildung No. 7 von Gerlach zu sehen ist, dass diese Kerne sich auch schon in den dickeren Nervenstämmchen des Grundplexus finden. Sie reichen weiterhin bis in die Scheidewandnerven hinein, die Kerne des „perimuskulären Netzes" sind demnach nur als letzte, am weitesten gegen die Muskulatur hin vorgeschobene Kerne der marklosen Nervenbündel überhaupt zu betrachten.

Auf Grund einer nochmaligen Durchsicht meiner früheren zablreichen Golgi- und Methylenblaupraparate und anf Grund neuer, absichtlich zur Prüfung dieser Sachlage hergestellter Methylenblaupräparate muss ich nun meine im Jahre 1902 aufgestellten, eben zitierten Behauptungen bezüglich der Bedeutung der Kerne an den "perimuskulären“ Nervengeflechten und des scheinbaren Netzes, welches an Gold-und mitunter auch an Methylenblaupräparaten durch diffuse Färbung des perimuskulären Plexus, dessen Bündeldann „durch spärliche mitgefărbte Teile des intramuskulären Plexus netzartig miteinander verbunden" erscheinen, in allem Wesentlichen aufrecht erhalten. Als perimuskulärer Plexus sind hierbei, wie schon bemerkt, bezeichnet dıe letzten, aus nur wenig Nervenfasern bestehenden, an die Muskelbündel heranziehenden Verzweigungen des Grundplexus. Über die Art dieser Aufzweigung des Grundplexus im Vorhofe orientiert am besten Abbildung 34 meiner Abhandlung in His' Arch. 1902, wo die längs der Muskelbündel absteigenden Abzweigungen der horizontal verlaufenden dickeren Nervenbündel dem perimuskulären Plexus von Gerlach entsprechen.

Zur weiteren Begründung meiner Stellungnahme gebe ich zunächst als Ergänzung zur Figur 37 meiner früheren Abhandlung in Parallelbildern (Figur 10 einerseits, 11 und 12 andererseits auf Tafel XXI) das Aussehen des Grundplexus bezw. seiner 
Aufzweigung bis zum perimuskulären Netz wieder, wie es sich in Methylenblaupräparaten darstellt. Die Abbildungen 10 und 11 sind aus dem Grundplexus der oberen Hohlvene entnommen, der sich mit Methylenblau leicht sehr schön färbt und ausserdem den Vorteil bietet, dass er im Flăchenpräparat mehr in einer Ebene liegt und nicht, wie zumeist im Vorhofe oder Ventrikel, sich auch stark nach der Tiefe zu erstreckt. Ich bemerke ausdrücklich, dass die hier erörterten Verbältnisse auch im Grund- und perimuskularen Plexus der Vorhofs- und Ventrikelmuskulatur wiederkehren (Fig. 12 stammt aus der Vorhofswand!), demnach in dieser Beziehung völlige Übereinstimmung zwischen den verschiedenen Herzabteilungen besteht. Die Unterschiede in der Anordnung des Grundplexus beziehen sich mehr auf die Art der Nervenverteilung (Genaueres darüber 1902, S. 93).

Betrachten wir nun zunächst Abbildung 10, so finden wir hier genau das schon von Gerlach beschriebene Bild eines mannigfach sich verzweigenden dicken violett gefärbten nervösen Stranges, in welchen von Strecke zu Strecke längliche oder mehr dreieckig geformte Kerne eingestreut sind. Verfolgt man diese Stränge und ibre Verzweigungen weiter, so anastomosieren sie deutlich miteinander und bilden also in der Tat ein Netzwerk, das grösstenteils auf der Muskulatur aufliegt. Sieht man sich diese Nervenstränge mit starker Vergrösserung genauer an, so entdeckt man freilich in ihrem Innern vielfache Ungleichmåssigkeiten der Färbung. Sie enthalten in mannigfachem Wechsel stärker gefärbte Körnchen, Fädchen, kugelige Konturen, die schon den Verdacht erwecken müssen, dass man es hier nicht mit einem einheitlichen, gleichmässig strukturierten Gebilde zu tun hat, sondern mit einem irgendwie komplizierter zusammengesetzten.

Wie diese Zusammensetzung beschaffen ist, ergibt sich nun aus der Betrachtung der Parallelfiguren 11 und 12. Hier ist keine Spur von diffuser Färbung des Gesamtplexus mehr (das kommt in der einfarbigen Zeichnung nicht so recht heraus!), vielmehr sondern sich scharf voneinander ab einzelne in Bündeln durcheinander geschlungene Fädchen, welche an den Teilungsstellen der Nervenbündel in verschiedener Richtung auseinanderweichen, eventuell mit anderen Faddchen aus demselben Bündel wieder zusammenlaufen, also einen $\mathrm{Pl}$ exus, kein anastomotisches Netz bilden, und welche neben sich ungefärbt längliche oder 
dreieckige Kerne liegen baben, dieselben Kerne, welche in der vorigen Figur 10 mitgefärbt waren. Wenn ich nun Bethe recht verstehe - und der Vergleich seiner Fig. 32 (Allgem. Anat. d. Nervensystems) mit meiner Fig. 11 bestärkt mich in dieser Meinung, trotzdem beide Abbildungen von verschiedenen Objekten herstammen - so würde er die letzte Figur so auffassen, dass hier die Neurofibrillen scharf herausdifferenziert sind, Kern und Protoplasma der Netzganglienzellen nicht mitgefärbt sind, während im ersteren Falle eine diffuse Färbung der Kerne, des Plasmas und der Fibrillen vorbanden wäre. Danach müsste also auch die Fig. 37 in Bethes Buch in seinem sinne so beurteilt werden, dass dort eine diffuse Färbung der Fibrillen, des Neuroplasmas und der Kerne stattgefunden hat. Dies ist nach Bethes Auffassung eigentlich ganz selbstverstandlich. Die Fig. 37 von Bet he gehört danach offenbar in dieselbe Reihe wie meine Fig. 10. Dies geht ausser aus allem anderen auch ohne weiteres aus der Beschreibung hervor, die Bethe von dieser Figur im Text gegeben hat. Bethe unterscheidet starkere Nervenfasern, welche sich sehr häufig mit Fasern, die von anderen Ganglienzellen ausgehen, verbinden und dünnere $Z$ weige, die vielfach nicht dicker als einzelne Neurofibrillen sind, welche nach Bethe augenscheinlich in die einzelnen Muskelfasern selbst eindringen sollen. "Dicke Nervenfasern" im perimuskulären Plexus gibt es aber nur in den Bildern der ersten Art mit diffuser Färbung, wie in Fig. 10, nie bei den Bildern zweiter Art mit der scharfen Differenzierung wie in den Fig. 11 und 12. Hier stellen sich auch bei ganz starker Vergrösserung die einzelnen Fädchen immer ganz ausserordentlich fein dar (vergl. z. B. auch Fig. 40 meiner Abhandlung vom Jahre 1902), nicht dicker als die allerfeinsten Fädchen, die Bethe in seinen Präparaten gesehen hat und die nach ihm nicht dicker sind als einzelne Neurofibrillen.

Es lässt sich nun mit aller gewünschten Klarbeit und Sicherheit der Nachweis erbringen, dass die in den Methylenblaupräparaten der beschriebenen zweiten Art gefärbten feinen Fådchen nicht einzelne, aus einer gemeinsamen Nervenfaser herausdifferenzierte Neurofibrillen sind, sondern in Wirklichkeit ganze Nervenfasern darstellen, welche zwar miteinander zu Bündeln vereinigt, im übrigen aber vollkommen selbständig und voneinander unabhängig sind. Dass diese Auffassung richtig ist, 
geht schon aus der Betrachtung der Methylenblaupräparate selbst deutlich hervor. Als Charakteristikum der Neurofibrillen wird stets betont (man vergl. insbesondere die Abhandlung ron Economo im Arch. f. Psychiatrie, Bd. 41, S. 158, 1906), dass sie vollkommen glatt sind. Nun sind zwar die blaugefärbten Fädchen der Methylenblaupräparate der zweiten Art streckenweise glatt, aber es kommen doch schon vereinzelte Varikositäten vor, und es finden sich in anderen Präparaten alle Übergänge von den glatten Fadchen zu immer reichlicheren Varikositäten, bis man schliesslich auf Bilder trifft, wo die Varikositäten eine nach der anderen dicht aufeinander folgen, ganz so, wie wir dies oben schon von den Nervenfasern der Chromatophoren festgestellt haben. Schon dort wurde betont, dass diese varikösen, meist bündelweise zusammęnliegenden Fădchen nicht differenzierte Neurofibrillen, sondern volle Nervenfasern darstellen, und ganz dasselbe gilt für die Herznerven.

Die Beobachtung dieser allmăhlichen Übergangsformen und der Vergleich mit den Chromatophorennerven weist nun aber auch den Weg, welcher zur Erklärung der s ch e inb a r einfachen Nervenfasern mit eingestreuten Kernen in den Methylenblaupräparaten der ersten Art führt. Ich habe in meiner früheren Abhandlung schon darauf hingewiesen, dass die Nervenfasern des perimuskulären, aber auch die des intramuskulären Plexus oft so dicht aneinander liegen, dass man sie auch mit der scharf differenzierenden Golg $\mathrm{i}$-Methode nicht voneinander sondern kann (ich verweise wieder auf Abbildung 32, 36 und 44 meiner früheren Abhandlung). Nun denke man sich ein solches Bündel von drei oder mehreren Nervenfasern sehr stark in Varikositäten zerfallen, etwa wie sie Ranvier in Fig. $249 \mathrm{~B}$ im technischen Lehrbuch der Histologie, S. 697, abbildet, so wird man völlig ausser Stande sein, in der breiten und fast gleichmässig gefärbten Masse die einzelnen Konstituenten zu unterscheiden.") Wir fanden ja ganz das Gleiche auch in den Chromatophorennerven! Offenbar wird dieBildung von Varikositäten durch die Prozeduren bei der Goldbehandlung sehr begünstigt, sodass man dabei in der Regel körnelige, diffus

1) Ich sehe bei diesem Hinweis ganz ab von der Nomenklatur R a nviers und verweise diesbezüglich auf die Darstellung von Tuckett (Journ. of Physiol., Bd. 19, S. 267, 1896), in welcher die Geschichte der Namen „R e maksche Fasern und Fibrillen" eingehend und klar auseinander gesetzt wird. 
gefärbte breite Massen erhält Auch bei der Methylenblaubehandlung kommt es sehr auf die spezielle Technik an. Ausdrücklich möchte ich bemerken, lass jene Methylenblaupräparate, in welchen eine solche diffuse Färbung dicker Nervenstränge vorhanden ist, sich bei der Durchsicht stets als die schlechteren („hässlicheren, unnatürlich aussehenden“) erweisen.

Noch viel schlagender als durch den Vergleich der Methylenblaupräparate untereinander ergibt sich aber die Richtigkeit meiner Auffassung auf einem anderen Wege. An allen guten Golgipräparaten lässt sich nåmlich nachweisen, dass der perimuskuläre Plexus von Gerlach aus Bündelchen isolierter Nervenfasern besteht. Zwar können sich diese streckenweise so dicht aneinander legen, dass man sie dort nicht gesondert wahrnehmen kann. Aber dass dies keine Verschmelzung ist, sieht man meist im weiteren Verlauf gelegentlich kleiner Biegungen oder Schlängelungen, wo dann die einzelnen Fäserchen, kleine Lücken zwischen sich lassend, auseinander treten können, so wie wir es auch an den entsprechenden Stellen der Chromatophorennerven gesehen haben. Nun wäre die Meinung an sich schon unhaltbar, dass bei den typischen Bildern der raschen Versilberungsmethode nach Golgi-Ra món bloss die Neurofibrillen imprägniert würden, vielmehr sind es immer die ganzen, oft ebenfalls varikösen Fasern. Ja noch mehr: ich besitze einige Präparate, und $z$ wei solche Stellen habe ich in meiner früheren Abhandlung abgebildet (Fig. 9 und 29) — wo der Übergang des Achsenzylinderfortsatzes der unipolaren Ganglienzellen des Froschberzens in den Grundplexus und weiterhin - wenigstens in Abbildung 9 bis zum intramuskulären Plexus in seiner ganzen Länge übersehen werden kann. Hier zeigt sich nun unwiderleglich, dass in der Tat jede einzelne imprägnierte Faser des Grundplexus dem geraden Fortsatze einer Ganglienzelle, also einer vollen Nervenfaser entspricht.

Schliesslich ist es mir neuerdings auch gelungen, den geraden Fortsatz einer Ganglienzelle aus dem obersten Abschnitte des $\checkmark$ entrikels mit Methylenblau zu färben und auf diese Weise mich noch überdies von der Übereinstimmung der Ergebnisse dieser Färbemethode mit denen der Golgimethode zu überzeugen. Die mit Methylenblau gefärbte Ganglienzelle mit ihrem geraden Fortsatze zeigte eine grosse Ähnlichkeit mit der in Fig. 13 meiner 
früheren Abhandlung abgebildeten versilberten Ganglienzelle. Ibr Achsenzylinderfortsatz lief namlich schwach gebogen nach rückwärts gegen die Atrioventrikulargrenze zu, was aber wahrscheinlich nur soviel bedeutet, dass er etwas weiter zentralwärts in einen Seitenast zur Muskulatur übergeht (vergl. S. 73 meiner früheren Abhandlung). Aufgefallen sind mir in diesem Präparate, sowie in einigen anderen die grossen Varikositäten, welche an den marklosen Nervenfasern im Ventrikel manchmal vorhanden sind, die aber doch wohl kaum mit kleinen Kernen verwechselt werden können.

Die soeben besprochenen Verhältnisse an Methylenblaupräparaten sind auch schon Smirnow ${ }^{1}$ ) aufgefallen und von ihm in folgender Weise beschrieben worden (1. c. $S .110 \mathrm{ff}$.) : „Das perimuskuläre und das intramuskuläre Netz L. Gerlach s erscheinen oft als solche Netze auch an den mit Methylenblan bearbeiteten Präparaten, nämlich bei mittlerer Vergrösserung; aber bei genauerer Analyse und bei der stärksten Vergrösserung einiger Präparate gelang es mir, mich davon zu überzeugen, dass wir es hier ebenfalls mit einem Geflecht und nicht mit einem Netz zu tun haben, und zwar: die Fibrillenbündel des Achsenzylinders einer Nervenfaser, welche sich von den uibrigen abgeteilt hat, gehen in eine andere Nervenfaser über, die nebenan liegt, oder sie durchkreuzen sich mit Bündeln von Nervenfibrillen einer anderen Faser. Deshalb muss man die scheinbaren oben genannten Netze L. Gerlachs für Geflechte von Fibrillenbündeln der Nervenfasern halten, d. h. für Geflechte der Achsenzylinderfibrillen. Es wäre sehr wichtig, festzustellen, ob wir es hier mit einem Geflecht aus den Primitivnervenfibrillen der Achsenzylinder einer und derselben Zelle oder mehrerer verschiedener Nervenzellen zu tun haben; das eine ist gleich wie das andere, meiner Ansicht nach, möglich." Die tatsächliche Beschreibung Smirnows stimmt, wenn man statt "Nervenfaser" das Wort "Nervenstrang" setzt, ganz mit dem äberein, was ich ebenfalls finde. Auch ist es sehr wohl möglich, dass sich die Achsenzylinderfortsätze der Ganglienzellen des Herzens bei ihren vielfachen Teilungen schliesslich in feinste Nervenfasern auflösen, welche ausser dem Neuroplasma nur noch eine einzige Neurofibrille enthalten, obwohl dies nach dem Gesagten noch von niemanden wirklich nachgewiesen wurde. Unrichtig wäre nur die Auffassung, dass diese feinsten - sagen wir aus nur e in er Neurofibrille samt dem zugehörigen Neuroplasma bestehenden Nervenfäserchen mit den ihnen anliegenden zu dickeren Nervenfasern verschmelzen, wie dies Smirnow anzunehmen scheint, was aber in Wirklichkeit nur durch die diffuse Färbung in den Präparaten der ersten Art vorgetäuscht wird.

Die Golgi-Präparate geben aber über unsere Frage noch eine weitere, sehr wichtige Aufklärung. Die mit dieser Methode

1) Zur Frage von der Endigung der motorischen Nerven in den Herzmuskeln der Wirbeltiere. Anat. Anz., Bd. 18. S. 105, 1900. 
im Grundplexus und seinen letzten Verästelungen (perimuskulärer Plexus Gerlachs) dargestellten Nervenfasern ziehen nämlich an den darin enthaltenen Kernen vorüber, ohne mit ihnen in Verbindung $z u$ treten, auch bei allerreichster Imprägnation der Fasern bleiben diese Kerne in der Regel gänzlich frei vom Niederschlag. Sie liegen demnach nicht in den Verlauf der Nervenfasern eingeschaltet, sondern neben ihnen. Als Belege dafür können die Fig. 29, 34 und 36 meiner früheren Abhandlung gelten, in denen trotz reichster Nervenfarbung nirgends ein Kern des perimuskulären Plexus imprägniert ist. Neu füge ich jetzt noch in Fig. 13 auf Taf. XXI eine Stelle hinzu, an der man einen solchen nicht imprägnierten Kern in einem Nervenbündel zwischen den imprägnierten, sich teilenden Nervenfasern liegen sieht. Die Analogie mit den Methylenblauprapparaten der Fig. 11 und 12 springt in die Augen. Die Kerne liegen in allen Fallen nicht in, sondern an den imprăgnierten oder gefärbten Nervenfasern. Freilich könnte es vorkommen, dass in manchen Golgipräparaten ausser den Nervenfasern gelegentlich auch diese Kerne mit imprägniert werden, so wie eben überhaupt ausser den nervösen Elementen alles mögliche andere sich mitunter imprägniert: Blutkörperchen, Bindegewebe, Endothelzellen, wohl auch die Kerne der bindegewebigen Kapsel der Ganglienzellen (vergl. S. 91 und die Stelle a der Fig. 15 in meiner früheren Abhandlung!) usf. Ich sage, sie könnten sich mit imprägnieren, denn sichere Beweisstellen dafür, wo dies gewiss der Fall ist, habe ich in meinen Präparaten nicht finden können. Es ist aber wohl möglich, dass sich hinter gewissen, auffällig dicken Varikositäten der Nervenfasern, die übrigens auch nicht häufig vorkommen, solche Stellen verbergen könnten, wo eine Mitinkrustation eines der Nervenfaser dicht anliegenden Kernes erfolgt war. Aber selbst wenn man dies annimmt, so bilden diese Stellen doch nur seltene und durch die Eigentümlichkeiten der Golgimethode leicht erklärbare Ausnahmen von der oben aufgestellten Regel, aus denen ein Rückschluss auf eine wirkliche Zusammengehörigkeit von Kern und Nervenfaser nicht gestattet wäre. Ganz und gar nicht aber dürfte man von einem Nervennetze sprechen, da diese Nervenfasern ja nicht miteinander anastomosieren. ${ }^{\mathbf{1}}$ ) Eine Stelle,

1) Dass die einzelnen Fasern des perimuskulären Plexus nicht miteinander anastomosieren, spricht ganz ausdrücklich auch schon Jacques 
wo ein Kern an einem Knotenpunkte des perimuskulären Geflechtes imprägniert wäre, findet sich in meinen zahlreichen Präparaten nirgends.

Mit dem so auf verschiedenen Wegen erbrachten Nachweis, dass die Kerne des perimuskulären Plexus mit den vorüberziehenden, mittels Methylenblau oder Silberchromat dargestellten Nervenfasern nicht zusammenhängen, făllt nun aber auch die Hypothese der Existenz von Nervennetzen im Herzmuskel wenigstens in der von Bethe aufgestellten Form. Freilich bestünde aber zunächst noch eine andere Möglichkeit. Es wăre denkbar, dass diese Kerne, welche den von den unipolaren Ganglienzellen des Froschberzens zur Muskulatur hinziehenden Nervenfasern anliegen, doch auch Kerne von Ganglienzellen sind, aber von solchen, welche sich mit Methylenblau und nach der Golgimethode gar nicht oder sehr schlecht färben, und deren Fortsätze vielleicht ein von den übrigen Nervenfasern unabhängiges Geflecht oder auch Netzwerk bilden. Ganz auszuschliessen ist diese Möglichkeit, mit Rücksicht auf das oben S. 393 erwähnte Verhalten der Methylenblaumethode, nicht. So hat ja neuerdings Carlson ${ }^{1}$ ) weitverzweigte Zellen aus dem Ventrikel des Salamanderherzens dargestellt, die er für Ganglienzellen bält und deren Fortsätze er in die benachbarten Nervenbündel übergehen sah. Es könnte sein, dass gerade das Herz des Salamanders ein günstiges Untersuchungsobjekt darbietet, bei welchem die Färbung dieser Zellen gelingt, während sie z. B. beim Frosch unmöglich ist. Es ist also gewiss zu verlangen, dass die Angaben von Carls on an seinem Objekt nachuntersucht werden. Dann wird auch zu diskutieren sein, in welcher Beziehung diese verzweigten Zellen Carlsons

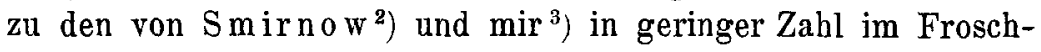
herzen gefundenen multipolaren Ganglienzellen stehen. Für unsere diesmaligen Überlegungen können wir aber davon ganz absehen. Denn selbst wenn sich die Annahme eines derartigen besonderen

aus (Recherches sur les nerfs du coeur chez la grenouille et les mammifères. Journ. de l'anat. et de la physiol., 1894, S. 635, zitiert in meiner früheren Abhandlung, S. 97.

1) Die Ganglienzellen des bulbus aortae und der Kammerspitze beim Salamander (Necturus macul.). Pflügers Arch., Bd. 109, S. 51, 1905.

2) Zur Kenntnis der Morphologie der sympathischen Ganglienzellen beim Frosche. Anat. Hefte, Bd. 14, S. 409, 1900.

3) His' Arch., 1902, S. $91 \mathrm{ff}$. 
Ganglienzellsystems im Herzen künftig bestätigen lassen sollte, ${ }^{\text {'}}$ ) so fiele dies keineswegs mit der Betheschen Nervennetzhypothese zusammen.

Dies geht ausser aus dem früher Gesagten noch aus folgendem hervor, was ich zur Klärung der Sachlage noch besonders hervorheben möchte. Bethe gibt an (auf S. 95 seines Buches), dass die Nervenfasern, welche die Bidderschen Ganglien an ihrem distalen Ende verlassen, in die Nervennetze der Kammer übergehen und zwar gewöhnlich bald in eine von den kleinen, dem Netze eigentümlichen Ganglienzellen, „deren Fortsätze zum Teil in die Muskulatur gehen, zum Teil mit den Fortsätzen anderer solcher Zellen Anastomosen eingehen." Nach Bethe sind also die Ganglienzellen des Netzes in den Weg der vom Bidderschen Ganglion zur Ventrikelmuskulatur hinziehenden Nervenfasern eingeschaltet, liegen nicht neben ihnen und bilden demnach kein für sich abgeschlossenes, unabhängiges Netz. Nun habe ich gleichfalls mehrmals die Ausstrahlung der Scheidewandnerven in den Ventrikel hinein nach Golgi-R a món imprägniert und auch hier dieselben Verhältnisse gefunden, wie sie oben S. 404 angegeben wurden, d. h. niemals sind bei Golgipräparaten neue Netzganglienzellen in den Verlauf dieser Nervenfasern eingeschaltet. Ja, ich besitze ein Präparat, in welchem ich direkt den geraden Fortsatz von Ganglienzellen in den Ventrikel hinein verfolgen kann, der sich dann im Ventrikel gerade so als selbständige Faser eines Nervenbündels darstellt, wie dies oben allgemein besprochen wurde. Übrigens stammt die Abbildung 39, die Bethe als Beleg für seine Angaben beifügt, allem Anschein nach aus einem Methylenblaupräparate mit distinkt gefärbten Nervenfasern, gehört also zu den oben so genannten "Bildern der zweiten Art“. Wie auch bei diesen der Anschein entstehen kann, dass die einzelnen Nervenfasern Auslaufer von Gerlachschen Kernen sind, das ist leicht zu verstehen, wenn man sich in meiner Fig. 12 den Kern intensiv mit Methylenblau gefärbt denkt. ${ }^{2}$ ) Es würde

1) Die physiologische Seite dieser Annahme, d. h. die Frage nach der Existenz eines von der Endausbreitung des Vagus und Accelerans gesonderten Nervennetzes für die Leitung der Erregung im Herzen, habe ich schon vor längerer Zeit in Pflügers Archiv (Bd. 72, S. 45̆ ff., 1898) ausführlicher erörtert.

3) $\mathrm{Ob}$ sich diese Kerne bei der Methylenblaubehandlung mitfärben oder nicht, hat man gar nicht in der Hand. In manchen Präparaten finden sich viele gefärbt, in manchen gar keine. 
dann geradezu unmöglich sein, zu entscheiden, ob die an den Kern herantretenden Nervenfasern sich, wie man bei fehlender Färbung desselben sieht, neben und über ihm durchkreuzen und verflechten, oder ob sie mit ihm verschmelzen. Übrigens dürften - soweit sich dies nach der blossen Abbildung ohne Kenntnisnahme des Präparates selbst beurteilen lässt - die ganz kleinen von $B$ ethe auch mit $z$ bezeichneten Stellen in seiner Fig. 39 wohl $\mathrm{zu}$ jenen schon von $\mathrm{Gerlach}$ erwähnten etwas grösseren, aber kernlosen Neuroplasmaanhäufungen an der Teilungsstelle von Servenfasern gehören (vergl. hierzu die Teilungsstelle b in meiner Fig. 12!).

Bei dieser Gelegenheit sei auch darauf hingewiesen, dass die Ganglienzellen des Bidder schen Ganglions denselben Bau und dieselbe Verbindung mit Nervenfasern aufweisen, wie die übrigen Ganglienzellen des Froschherzens, und dass sich keinerlei Nachweis dafür erbringen lässt, dass sie zwischen das "Nervennetz" der Vorkammer und der Kammer zwischengeschaltet sind. Das intramuskuläre Nervengeflecht der Vorkammern geht vielmehr mitsamt der Muskulatur $\mathrm{n}$ eben den Bidderschen Ganglien ohne Unterbrechung von den Vorhöfen auf die Kammer über, wie ich seinerzeit ${ }^{1}$ ) vielleicht etwas zu kurz, aber nachdrücklich genug, erwähnt habe. Wie Bethe (l. c. S. 94) zu der entgegengesetzten Meinung kommen konnte, ist mir unklar.

Wenn nun nach dem Gesagten zwar die Möglichkeit, dass unter den Kernen des perimuskularren Plexus vielleicht auch einige Ganglienzellkerne enthalten sind, nicht unbedingt ausgeschlossen werden kann, so ist es doch zum mindesten höchst unwahrscheinlich, dass diese Annahme für alle solchen Kerne gelten sollte. Ich will dabei ganz absehen davon, dass ja, wie bereits Gerlach anführt, ihr "Habitus" dieser Annahme Schwierigkeiten macht, sondern möchte nur nochmals darauf hinweisen, dass sich Kerne in voller Kontinuität vom perimuskulären Plexus an zurück in die dickeren Stämmchen des Grundplexus und in die Scheidewandnerven binein reichlich vorfinden. Wir stossen daher auch hier wieder auf dieselben Verhältnisse, wie wir sie ganz ebenso schon bei den Chromatophorennerven beschrieben haben, und wie sie sich überhaupt an allen marklosen Nervenbündeln, insbesondere den postganglionären sympathischen Nervenfasern vor-

1) Pflügers Arch., Bd. 72, S. 455, 1898; His' Arch., 1902, S. 103. Die an der letzteren Stelle gemachte Einschränkung bezieht sich bloss auf die unentschiedene Frage, ob ein kontinuierliches $\mathrm{N}$ etz oder ein blosses Nervengeflecht anzunehmen sei. 
finden. Wir müssen daher, wenn wir die Frage nach der Natur dieser Kerne im Herzen beantworten wollen, ganz allgemein die Verbältnisse bei den postganglionăren sympathischen Nervenfasern ins Auge fassen. Hier geben uns nun zunächst Aufklärung die Untersuchungen von Tuckett. ${ }^{1}$ ) Dieser fand die einzelnen marklosen postganglionären Nervenfasern (z. B. vom Ganglion cervicale superius) umgeben von einer Scheide, in welche Kerne eingelagert waren, und er vermutet, dass diese Scheide eine Fortsetzung der Kapsel sei, welche die sympathischen Ganglienzellen umgibt. Mit der Methylenblaumethode hatte kurz vorher A. S. Dogiel ${ }^{2}$ ) in der Gallenblase von Saugetieren an den Achsenzylinderfortsätzen der sympathischen Nervenfasern ovale Kerne dargestellt. Zuweilen konnte er beobachten, dass ein solcher Kern von einer feinen Protoplasmaschicht umgeben war, welche sich an den Polen des Kernes in einen feinen Fortsatz in die Lange zog, sodass das ganze Gebilde eine spindelförmige Gestalt erhielt, d. h. in der Tat als eine Zelle erschien. Ob diese spindelförmigen Zellen zur Schwannschen Scheide gehören, oder ob sie dem Achsenzylinderfortsatze selbst unmittelbar aufliegen, das liess er unentschieden. Es ist nun eigentlich ganz selbstverständlich, dass man solche dicht an einzelne n Nervenfasern anliegende Kerne auch im Grundplexus der Herzmuskulatur bei der Methylenblaufärbung findet, wo sie dann oft etwas intensiver gefärbt sind und mit ihrer Nervenfaser in e in Gebilde verschmelzen. Fraglich aber ist es, ob man die Kerne des perimuskulären Plexus alle hierher rechnen kann. Diese erscheinen - wenigstens $\mathrm{zu}$ einem Teile - doch wohl zu gross, als dass sie nur einer daneben hinziehenden Nervenfaser angehören könnten und ich halte es daher auch heute noch für richtiger, diese grösseren Kerne als Kerne der bindegewebigen Hülle des ganzen Nervenbündels, nicht bloss einer einzelnen Faser daraus, zu deuten. Indessen möchte ich mich in diesem speziellen Punkt jetzt doch etwas zurïckhaltender aussprechen, als in meiner früheren Abhandlung. Denn es ist zu bemerken, dass auch die von A. S. Dogie 1 in der oben zitierten Abhandlung abgebildeten

1) On the structure and degeneration of non medullated nerve fibres. Journal of Physiol., Bd. 19, S. 267, 1896. Hier auch die frühere Literatur darüber.

2) Zur Frage iiber den feineren Bau des sympathischen Nervensystems bei den Säugetieren. Arch. f. mikr. Anat., Bd. 46, S. 311 und 394 ff. 1895. 
Kerne oft sehr beträchtlich breiter sind als die zugehörigen feinen Nervenfasern. Ich möchte es daher heute bloss als wahrscheinlich bezeichnen, dass unter den Kernen der marklosen Nervenbündel des Grundplexus zweierlei Arten zu unterscheiden sind: solche, welche nur einzelne Nervenfasern umscheiden (Dogiel, Tuckett), und solche, welche der bindegewebigen Hülle des ganzen Nervenbündels angehören.

$O b$ auf den Onterschied, welchen die Kerne der Figuren 10 und 12 einerseits und der Figuren 11 und 13 andererseits zeigen - die ersteren sind bomogen, die letzteren fein granuliert - ein besonderes Gewicht zu legen ist, vermag ich vorläufig noch nicht anzugeben.

Es wăre schliesslich noch meine Aufgabe, auf die Frage der Nervennetze in den Blutgefässen einzugehen, doch kann ich mich darüber ganz kurz fassen. Erstens müssen wir nämlich schon von vornherein voraussetzen, dass die Verhältnisse an den Gefässen ähnlich liegen, wie am Herzen, und gar die oberen Hohlvenen, von denen unsere Figuren 10 und 11 berstammen, müssen wir ja schon zu den Gefässen selbst rechnen. Andererseits wurden im letzten Jahre in meinem Laboratorium Versuche angestellt, welche vorläufig ergaben, dass nach Durchschneidung der postganglionären sympathischen Gefässnerven für eine bestimmte Region sich in der Gefässwand dieses Gebietes mit der Methylenblaumethode nur noch spärliche Reste von Nervengeflechten nachweisen lassen, dass sie im übrigen, wie sich durch anderweitige Beobachtungen noch mehr erhärten liess, degenerieren. Hier liegt also offenbar kein selbständiges peripheres Ganglienzellsystem vor, - was natürlich auch wieder einen Wahrscheinlichkeitsrückschluss auf das Herz gestattet. Leider wird sich die Veröffentlichung dieser Untersuchung aus äusseren Gründen noch einige Zeit hinausziehen. Während dieses Problem bearbeitet wurde, erschien eine Abhandlung von Lapinsky, ${ }^{1}$ ) welcher zu demselben Resultate kam. ${ }^{2}$ ) Derselbe Autor hatte übrigens schon

1) Zur Frage über die Beteiligung der Nervenstämme der hinteren Extremität an der vasomotorischen Innervation der distalen Gebiete derselben und über die Veränderung der vasomotorischen Elemente sowie der Gefässe selbst der Hinterpfote nach Beschädigung des nervus ischiadicus. Virchows Arch., Bd. 183, S. 1, 1906.

2) Wenn hier von zwei Seiten zu gleicher Zeit und unabhängig voneinander dasselbe Resultat gefunden wurde, so kann das wohl als eine erfreuliche gegenseitige Bestätigung bezeichnet werden. 
in einer vorausgehenden Abhandlung ${ }^{1}$ ) seine Meinung über die Kerne der Nervennetze an den Blutgefässen dahin geäussert, dass hier keine Ganglienzellen, sondern Kerne einer strukturlosen Nervenscheide vorliegen. Ich muss mich aus den angegebenen Gründen jetzt ebenfalls in gleichem Sinne aussprechen, und benütze diese Gelegenheit, um eine irrtümliche Meinung, die ich in Nagels Handbuch der Physiologie des Menschen ${ }^{2}$ ) ausgesprochen habe, zurückzunehmen. Ich hatte geglaubt, dass die von Bethe angenommenen Ganglienzellen identisch seien mit den von verschiedenen Autoren beschriebenen, bald als Ganglienzellen, bald als Bindegewebszellen bezeichneten, verzweigten Zellen, welche von S. Mayer ${ }^{3}$ ) zum Teil als verzweigte glatte Muskelzellen gedeutet werden. Nachdem ich aber jetzt diese Zellen aus eigener Erfahrung kennen gelernt habe, muss ich diese Äusserung zurücknehmen. Die Rouget-Mayerschen Zellen sind viel kleiner als die sogenannten „Ganglienzellen" der Nervennetze von Bethe, sodass sie mit ihnen kaum verwechselt werden können. Es kann sich daher bei den Kernen der' Nervengeflechte an den Gefassen auch wieder nur um Kerne von Nervenhüllen handeln. Ob es eigentliche Bindegewebszellen oder Kerne einer Schwannschen Scheide sind, lasse ich dahingestellt.

\section{Résumé.}

Wenn wir die oben dargelegten eigenen Untersuchungen mit denen der anderen Autoren zusammenfassen, so finden wir in der gesamten glatten und der ihr verwandten Muskulatur der Wirbeltiere und Cephalopoden gemeinschaftliche, überall wiederkebrende Innervationsverhältnisse: Aus den zur Muskulatur hinziehenden Nervenbündeln bildet sich durch Abschwenkungen und Teilungen der in ihnen enthaltenen gröberen Nervenfasern zunăchst ein Nervengeflecht, der Grundplexus, der insbesondere in der Nahe der Eintrittsstelle der Nervenbündel stark entwickelt ist, dann sich aber immer feiner aufteilt, sodass er schliesslich

1) Die Gefässinnervation der Hundepfote. Dies Arch., Bd. 65, S. 623, 190ら. Hier ist auch die frühere Literatur angegeben.

2) Bd. I, S. 307, unten.

3) Die Muskularisierung der kapillaren Blutgefässe. Nachweis des anatomischen Substrats ihrer Kontraktilität. Anatom. Anzeiger, Bd. 21, S. 442, 1902. 
nur noch aus wenigen nebeneinander laufenden Nervenfasern besteht, der aber vor allem dadurch charakterisiert ist, dass er von der Verlaufsrichtung der Muskelzüge unabhängig ist, oft geradezu quer über sie hinwegzieht. Von diesem Grundplexus gehen kürzere oder längere Verbindungsstücke ab (etwa dem perimuskulären Plexus von Gerlach und dem intermediären Plexus von Klebs entsprechend, die aber als allmăhlicher Übergang vom Grundplexus zum Endplexus kaum als gesonderte Plexus bezeichnet werden können) zu einem Geflecht, dem Endplexus, dessen einzelne Nervenfädchen längere Strecken ganz dicht an den Muskelzellen, welche die Muskelbündel oder Muskelschichten konstituieren, hinziehen und infolgedessen die Anordnung der Muskulatur sehr genau wiedergeben. Wo die Nervenfärbung möglichst vollständig gelingt, findet man, dass im Endplexus die Nervenfädchen ohne Aufhören sich fortwährend weiter verfolgen lassen, freie Nervenenden also nicht vorhanden sind (Herzmuskel der Amphibien bei der Golgi-Imprägnation) oder die Nervenfasern an die Gegend des Muskelkernes herantreten und dann, ohne zu endigen, schleifenförmig umbiegen und von dem innervierten Muskel wieder wegziehen (Chromatophorenmuskeln). Daraus folgt, dass die bei unvollständiger Färbung beobachteten sogenannten knöpfchenförmigen freien Enden dieser Nervenfasern Kunstprodukte sind, dass in Wirklichkeit vielmehr mindestens die Teiläste einer jeden einzelnen zur Muskulatur hinziehenden Nervenfaser unter sich ein wahres Endnetz bilden. Ob solche netzförmige Verbindungen auch zwischen Nervenfasern verschiedener Herkunft bestehen, ob also ein kontinuierlich durchgehendes Endnetz in diesen Muskeln vorhanden ist, liess sich nicht entscheiden. Wenn aber ein solches kontinuierliches Nervennetz wirklich bestehen sollte, so wird es von den Fädchen des Endplexus gebildet, die Fasern des Grundplexus anastomosieren nicht miteinander. Spezifische Nervennetze im Betheschen Sinne, die aus breit anastomosierenden Ganglienzellen gebildet werden sollen, sind in der untersuchten Muskulatur nicht vorbanden. Die Bethe schen

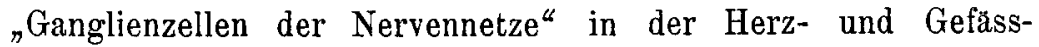
muskulatur der Wirbeltiere sind höchst wahrscheinlich Kerne von Nervenhüllen, welche bei manchen Untersuchungsmethoden (in Gold- und manchen Methylenblaupräparaten) mit den vorüberziehenden Nervenfasern zu einer gemeinschaftlichen Masse ver- 
schmolzen erscheinen, während man mit anderen Methoden (Methylenblau in gewissen Fällen, insbesondere aber mit der Golgimethode) ganz sicher nachweisen kann, dass die Nervenfasern mit diesen Kernen in keinem Zusammenhang stehen.

\section{Erklärung der Abbildungen auf Tafel XXI.}

Die Fig. 1-4 und Fig. 7 sind von A. Kirchner (Leipzig), die Fig. 5, 6, 8-13 von meinem Assistenten Herrn Dr. Eugling gezeichnet worden.

Fig. 1. Fünf nebeneinander liegende, verschieden grosse $\mathrm{Ch}$ romatophoren aus der Haut von Loligo vulgaris mit sich gegenseitig überkreuzenden Radiärmuskelfasern. Die letzteren und ihre Kerne mit Methylenblau gefärbt. Fixierung mit Ammonpikrat. Zeiss' Obj. A. Beschreibung im Text S. $367 \mathrm{ff}$.

Fig. 2. Nervengeflecht aus der Chromatophorenschicht der Haut von Loligo vulgaris. Alles Gezeichnete, bis auf die dunklen Flecken, welche Pigmentkörper von Chromatophoren darstellen, sind mit Methylenblau gefärbte Nervenbündel. Die Nervenbündel $\mathbf{a}, \mathbf{b}$ und $\mathbf{c}$ ziehen entlang von Radiärmuskeln $z u$ einer nicht gezeichneten Chromatophore. Zeiss' Obj. A. Beschreibung im Text S. 370, 373 ff., 381.

Fig. 3. Verästelung einer breiten Nervenfaser des Grundplexus bis in die Nervenzweige za den Chromatophoren. Loligo vulgaris. a Stammnervenfaser. b, c, d Bündelchen des Grundplexus mit sehr wenigen gefärbten Nervenfasern. e stark geschlängelter Nervenzug an einer kontrahierten (abgerissenen?) Radiärfaser. f, g Nervenfasern, welche zur rechtsgezeichneten Chromatophore hinziehen. Der Nerv h zieht über die rechte Chromatophore hinweg. Methylenblaufärbung; Fixierung mit Ammonpikrat. Gezeichnet mit Zeiss' D, Ok. 3, nachkontrolliert mit Immersion. Beschreibung im Text S. $371,381$.

Fig. 4. Guirlandenförmige Nervenschlingen von einer Radiärfaser zur anderen in der Nähe des Chromatophorenkörpers. Loligo vulgaris. Färbung etwas diffus, sodass der Eindruck eines einheitlichen blassen Nervenstranges mit schärfer tingierten Fädchen zustande kommt. a, b Nerrenschlingen, welche von der Guirlande abgehen und auf der Oberfläche des Muskels sich ausbreiten. c, d, f, g scheinbare freie Enden von Nervenfasern. Vergl. dazu die Beschreibung im Text S. 375, 377, 379. Methylenblau, Ammonpikrat. Gezeichnet mit Zeiss' Immers. 1,30, Komp.-Ok. 4, etwas verkleinert.

Fig. ó und 6. Zwei Stellen eines Bündels von Nervenfasern, welche an einer Radiärfaser entlang laufen und sich in Fig. 5 nicht voneinander sondern lassen, in Fig. 6 aber auseinander treten. 
Schärfere Tingierung der Nervenfasern als in Fig. 4. Das Ende $x$ der Fig. 6 ist gegen die Stelle der Fig. $\bar{a}$ (und den Pigmentkörper der Chromatophore) hin gerichtet. Methylenblau, Ammonpikrat. Gezeichnet mit Zeiss' hom. Immers. 1,30, Komp.-Ok. 4, kontrolliert mit Komp.-0k. 12 und 18. Beschreibung im Text S. $375 \mathrm{ff}$.

Fig. 7. Nervengeflecht der Flossenmuskulatur. Sepia elegans. Aufsicht auf zwei Längsmuskelschichten A und B; letztere gibt den schrägen Zug $\mathrm{C}$ ab. D tiefere Partie der Längsschicht $B$. E Aufsicht auf die Transversalmuskelschicht, a, b, c Nervenfasern. Genauere Beschreibung im Text S. 384. Methylenblau, Ammonpikrat. Zeiss' D, Ok. 3.

Fig. 8. Nervenscheide mit Kern an einer marklosen Nervenfaser von Loligo vulgaris. a Nervenscheide, b Kern derselben, c Teilung der Nervenfaser, der dünnere Teilast intensiver gefärbt als der andere, d Knickung der Nervenfaser. Methylenblan-Ammonpikrat. Zeiss hom. Immers. 1,30, Komp.-0k. 4. Beschreibung im Text S. 391.

Fig. 9. Unipolare periphere Ganglienzelle in der Haut von Loligo vulgaris, in einem Nervenbündel liegend. Methylenblau, Ammonpikrat. Zeiss' hom. Imm. 1,30, Komp.-0k. 4.

Fig. 10. Nervenstrang des "perimuskulären" Plexus mit Kernen von der oberen Hohlvene des Froschherzens. Methylenblau, Ammonpikrat. Zeiss' homogene Immers. 1,30, Komp.-Ok. 4. Beschreibung im Text S. $398 \mathrm{ff}$.

Fig. 11. Nervenbündel des Grundplexus mit Kern von der oberen Hohlvene des Froschherzens. Methylenblau, AmmonmolybdatOsmiums. Zeiss' hom. Immers. 1,30, Komp.-Ok. 4. Beschreibung im Text S. 398 ff.

Fig. 12. Nervenbündel des perimuskulären Plexus mit Kern aus der Vorhofswand des Froschherzens. Methylenblau, AmmonmolybdatOsmiumsäure. Zeiss' hom. Immers. 1,30, Komp.-Ok. 4. Beschreibung im Text S. 398 ff.

Fig. 13. Nervenfasern aus dem Grundplexus der Vorhofscheidewand. Golgipräparat mit nicht imprägniertem Kern bei a. b Teilungsstelle einer Nervenfaser mit besonders grosser Varikosität. Zeiss' hom. Immers. 1,30, Komp.-Ok. 4, Beschreibung im Text S. 404. 